\title{
Kalman filter tracking and vertexing in a silicon detector for neutrino physics.
}

\author{
A. Cervera-Villanueva ${ }^{1,2}$, E. do Couto e Silva ${ }^{1,3}$, M. Ellis ${ }^{4,5}$, D. Ferrère ${ }^{1,6}$, J.J. Gómez-Cadenas ${ }^{1,2}$, \\ M. Gouanère ${ }^{7}$, J.A. Hernando ${ }^{2,8}$, J. Kokkonen ${ }^{1}$, V.E. Kuznetsov ${ }^{1,9,10}$, L. Linssen ${ }^{1}$, Ö. Runolfsson ${ }^{1}$, \\ F.J.P. Soler ${ }^{1,4,11}$.
}

\begin{abstract}
This article describes the application of Kalman filter techniques for the tracking and vertexing of particles inside the NOMAD-STAR detector, a silicon vertex detector installed in NOMAD, one of the neutrino oscillation experiments at the CERN-SPS. The use of the Kalman filter simplifies computationally the tracking and vertex procedure for NOMAD-STAR. The alignment of NOMAD-STAR is shown as an example of the application of the Kalman filter for tracking purposes. The accuracy of the method is such that one obtains alignment residuals between 9 and $12 \mu \mathrm{m}$. Furthermore, a preliminary measure of the impact parameter (with an RMS $\sim 36 \mu \mathrm{m}$ ) illustrates the vertexing capabilities of this technique.
\end{abstract}

Accepted for publication in Nuclear Instruments and Methods in Physics Research A

\footnotetext{
${ }^{1}$ CERN, Geneva, Switzerland.

${ }^{2}$ IFIC, Universidad de Valencia, Spain.

${ }^{3}$ Now at Stanford Linear Accelerator Center, USA.

${ }^{4}$ University of Sydney, Sydney, Australia.

${ }^{5}$ Now at Rutherford-Appleton Laboratory, UK.

${ }^{6}$ Now at the Université de Genève, Geneva, Switzerland.

${ }^{7}$ LAPP, Annecy, France.

${ }^{8}$ Now at Universidad de Santiago de Compostela, Spain.

${ }^{9}$ Joint Institute for Nuclear Research, Dubna, Russia.

${ }^{10}$ Now at Fermi National Laboratory, USA.

${ }^{11}$ Now at University of Glasgow and Rutherford-Appleton Laboratory, UK.
} 


\section{Introduction}

There is great interest in the study of neutrino oscillations as a means to understand whether neutrinos have mass. Two experiments at CERN, CHORUS and NOMAD [1, 2], have been searching for exclusive $\nu_{\mu}\left(\nu_{e}\right) \leftrightarrow \nu_{\tau}$ oscillations by two different methods. CHORUS searches for the $\tau$ decay topology inside an emulsion target, while NOMAD searches for the kinematical signature of the $\tau$ decays inside a light drift chamber target. It has been proposed that future $\nu_{\mu}\left(\nu_{e}\right) \leftrightarrow \nu_{\tau}$ searches could benefit from the use of a large surface silicon tracker, either in conjunction with a passive target or with an emulsion target, to enhance the $\tau$ detection capabilities $[3,4,5]$. The selection of the different $\tau$ decay candidates in this case is by means of an impact parameter or a double vertex signature. As a means to test the feasibility of detecting $\nu_{\tau}$ s by the use of silicon microstrip detectors, we built an instrumented Silicon TARget (NOMAD-STAR), which was installed in the NOMAD spectrometer at the beginning of 1997 (Fig. 1).



Figure 1: The NOMAD detector with the Silicon TARget (NOMAD-STAR).

The NOMAD-STAR detector, shown in Fig. 2, was installed upstream of the first NOMAD drift chamber. It consists of four layers of boron carbide $\left(\mathrm{B}_{4} \mathrm{C}\right)$ of dimensions $72 \times$ $31.5 \times 2.0 \mathrm{~cm}^{3}$, amounting to a mass of $45 \mathrm{~kg}$ (density of $2.49 \mathrm{~g} \mathrm{~cm}^{-3}$ and radiation length 
$X_{0}=21.9 \mathrm{~cm}$ ), interleaved with layers of single-sided silicon microstrip detectors (manufactured by Hamamatsu Photonics, Japan), with an additional layer of silicon detectors downstream for better track reconstruction. The five layers of silicon detectors have an active surface of $1.14 \mathrm{~m}^{2}$ and consist of 10 overlapping ladders, with 12 silicon microstrip detectors per ladder, read out by low-noise VA1 chips [6]. The detectors are AC coupled, FOXFET biased [7], passivated with silicon oxide and consist of 641 readout strips (with a pitch of $50 \mu \mathrm{m}), 640$ of which are read out by the electronics. The strips are oriented parallel to the NOMAD magnetic field ( $x$ axis in Fig. 1). The performance of the silicon ladders has been described in [8], where a beam of pions with momenta higher than $100 \mathrm{GeV} / \mathrm{c}$ was used to determine that the point resolution of a ladder of 12 detectors is about $5 \mu \mathrm{m}$. A general description of the NOMAD-STAR detector can be found in [9].

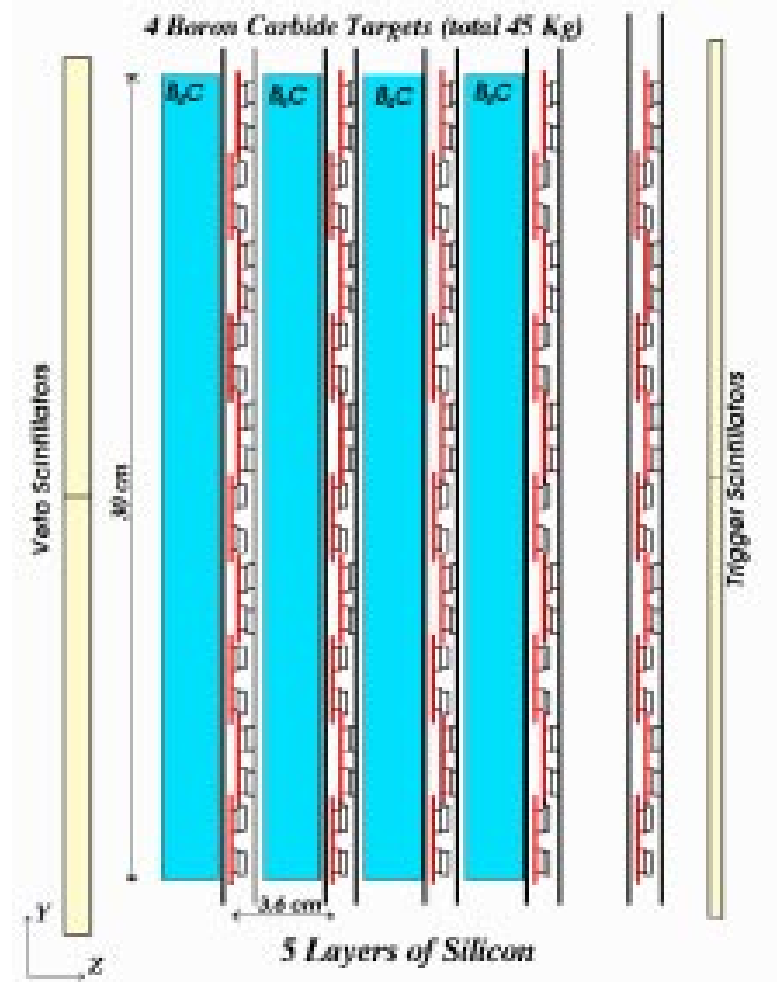

Figure 2: Schematic of the side view of the silicon target.

The present paper will describe the application of Kalman filtering techniques [10] for the reconstruction of tracks inside the NOMAD-STAR detector and its matching to tracks inside the drift chamber target of NOMAD. It commences with a statement of the problem of reconstructing tracks and a motivation of the Kalman filter as an optimal solution to this problem (section 2). A general description of the Kalman filter model used for tracking and vertexing in NOMAD-STAR is described in section 3. The alignment procedure of NOMADSTAR is used as an example of Kalman filter tracking in section 4, while a first estimate of 
the impact parameter resolution of the silicon detector is used in section 5 as an application of the Kalman filter vertexing.

\section{Motivation}

Like every other neutrino detector, NOMAD-STAR has to achieve two contradictory goals: produce a large number of neutrino interactions, which requires a mass as large as possible, and measure the products of these interactions with the maximum precision, which would imply minimising the material budget. To separate a putative $\nu_{\tau}$ interaction from the bulk of $\nu_{\mu}$ charged current (CC) events, NOMAD-STAR takes advantage that the $\tau$ produced in a $\nu_{\tau}$ interaction has a relatively long lifetime $(c \tau=86.93 \mu \mathrm{m})$, which results in an impact parameter distribution (see Fig 3) that is larger, on average, than the impact parameter distribution of $\nu_{\mu}$ events. The impact parameter $(d)$ is defined as the projected signed distance of the closest approach of the $\mu^{-}$from a $\nu_{\mu}$ CC interaction (or the decay track from the one-prong decay of a $\tau$ in the case of a $\nu_{\tau} \mathrm{CC}$ interaction) to the vertex produced by the remaining hadronic jet. One can also define the projection of the impact parameter onto the y-coordinate $\left(d_{y}\right)$.

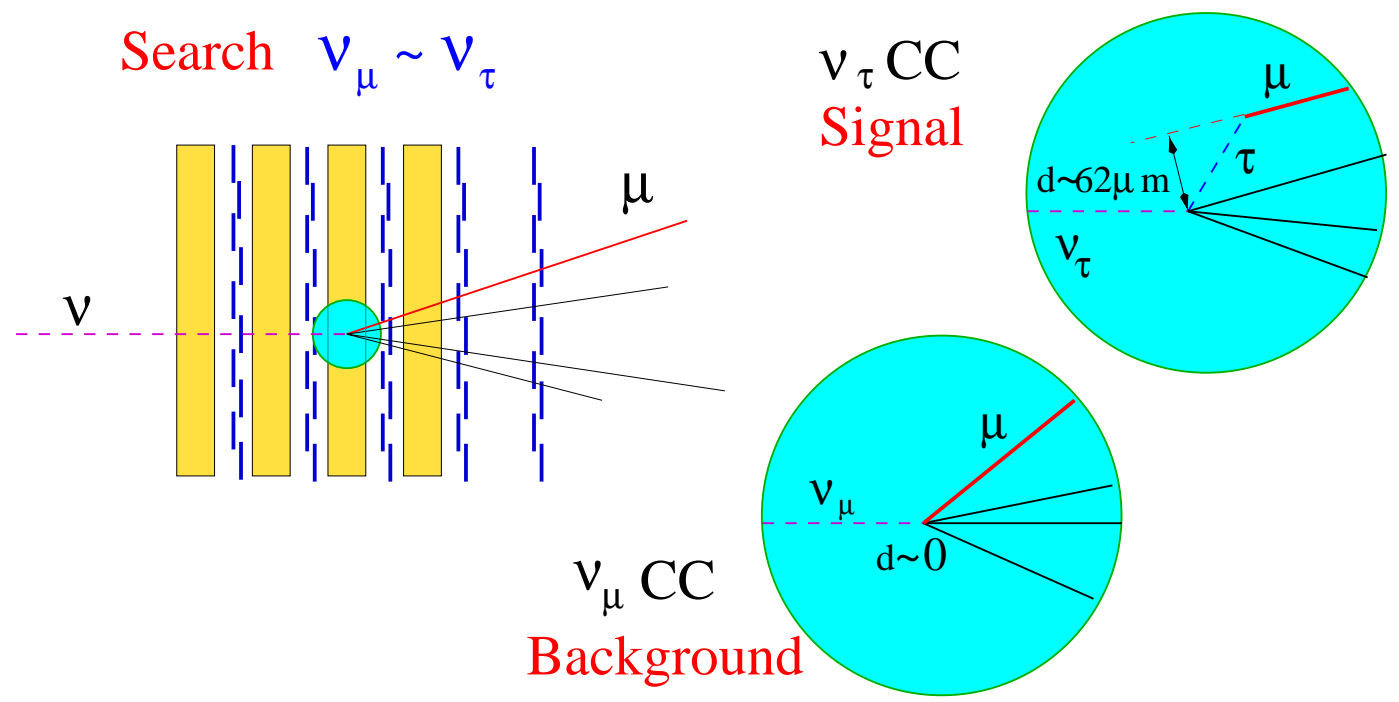

Figure 3: Definition of the impact parameter.

NAUSICAA [3] was a detector concept for a $\nu_{\tau}$ appearance search, with a configuration that was very similar to NOMAD-STAR, consisting of layers of silicon strip detectors $(50 \mu \mathrm{m}$ readout pitch) interleaved with $2.2 \mathrm{~cm}$ thick layers of a passive graphite target with a total mass between 1-2 tons. A neutrino beam similar to the one at the CERN-SPS served to simulate $\nu_{\mu}$ and $\nu_{\tau}$ events inside the detector. Fig. 4 (from [3]) shows the expected projected impact parameter distributions (and impact parameter significance $d_{s} \equiv d_{y} / \sigma_{y}$ distributions) 
of $\nu_{\mu}$ and $\nu_{\tau} \mathrm{CC}$ events obtained for NAUSICAA. The projected impact parameter for the $\nu_{\tau}$ signal events is $\sim 62 \mu \mathrm{m}$, while the impact parameter for the background $\nu_{\mu} \mathrm{CC}$ interactions, which is only due to measurement errors, is centered at zero and has a RMS of $\sim 28 \mu \mathrm{m}$.
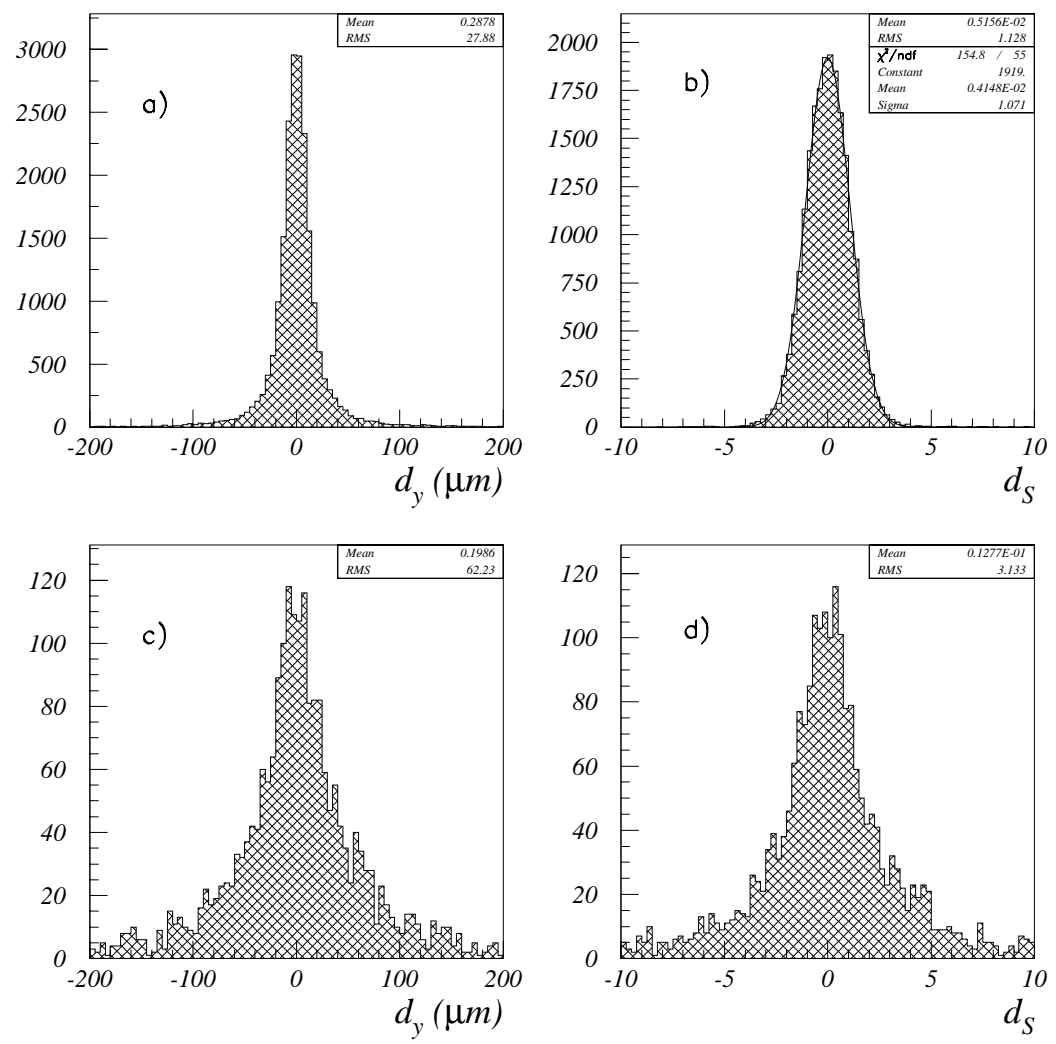

Figure 4: Projected impact parameter $\left(d_{y}\right)$ and impact parameter significance $\left(d_{s} \equiv d_{y} / \sigma_{y}\right.$ for a) and b) $\nu_{\mu} \mathrm{CC}$ interactions, and c) and d) $\nu_{\tau} \mathrm{CC}$ interactions.

Since particles traversing NOMAD-STAR may cross several layers (with each $\mathrm{B}_{4} \mathrm{C}$ layer containing $\sim 10 \%$ of a radiation length $X_{0}$ and each silicon layer $\sim 4 \% X_{0}$ ), the effect of Coulomb multiple scattering is very important. The design of the detector is such that the measurement error of the average track $(\sim 10 \mu \mathrm{m})$ is of the same order as the error induced by multiple scattering. On the other hand, multiple scattering induces an unavoidable correlation between the measurements in different planes. The covariance matrix of the measurements is no longer diagonal. Consequently, to obtain the fit parameters via a $\chi^{2}$ minimisation, one has to invert a matrix of dimension $N^{3}$, where $N$ is the number of measurements. Furthermore, since the track model is not linear, one has to deal with a non-linear least-squares fit, which often requires a laborious and not always robust iterative 
procedure.

Fortunately, as demonstrated by Frühwirth [11] and widely applied to high energy physics processes, [12] to [20], (including the general NOMAD reconstruction [21]), the use of robust techniques, such as the Kalman filter [10], allows one to address the case in which the covariance matrix of the measurements is not diagonal. Basically, what the Kalman filter does is a) to propagate the parameters and their covariance matrix from one measurement plane to the next using the track model, while transporting the noise matrix including the errors induced by multiple scattering, energy losses and other random processes (prediction), b) compute the parameters by taking the weighted mean of the propagated values and the actual measurements in that plane (filtering) and c) update the fits by including the new information (smoothing). The Kalman filter only requires inversions of $N \times N$ matrices.

Our problem can be further simplified by the realization that the track model for the case of NOMAD-STAR can be linearised without loss of accuracy. A linearised model permits a simple, intuitive and elegant formulation of the problem.

\section{The Kalman filter for track and vertex fitting}

\subsection{Background}

The Kalman filter is the optimal estimator of the state vector of a linear dynamical system, since it minimizes the mean square estimation error $[10,11]$. A track in space can be described by its 5 -dimensional state vector, which can be parametrized as follows $\mathbf{x}=$ $(x, y, d x / d z, d y / d z, 1 / p)$, where $x, y$ and $z$ are the spatial coordinates and $p$ is its momentum, at each of the measurement points, defined by their $z$ coordinate along its trajectory. In its linear form, the evolution of the state vector is described by the discrete system of linear equations:

$$
\mathbf{x}\left(z_{k}\right) \equiv \mathbf{x}_{k}=\mathbf{F}_{k-1} \mathbf{x}_{k-1}+\omega_{k-1}
$$

which defines the change in status of this vector based on the previous measurement point $\mathbf{x}_{k-1}$. The matrix $\mathbf{F}_{k-1}$ is the track propagator from measurement $k-1$ to measurement $k$ and the random variable $\omega_{k-1}$ describes the random noise of the system (also called process noise). In the tracking of particles through dense media, the process noise can be due to multiple scattering, energy loss or any other physical process that might disturb the particle trajectory.

The actual measurements $\mathbf{m}_{k}$ carried out at each of the measurement points are a function of the state vector:

$$
\mathbf{m}_{k}=\mathbf{H}_{k} \mathbf{x}_{k}+\epsilon_{k}
$$

where $\mathbf{H}_{k}$ describes the relationship between the measured quantities and the state vector and $\epsilon_{k}$ describes the measurement noise.

The Kalman filter proceeds by performing these three distinct operations: 
- Prediction, where the status of the state vector is estimated at a future measurement point;

- Filtering, where the current estimation of the state vector is carried out based on the previous measurements; and

- Smoothing, where the estimation of the state vector at a previous measurement is re-evaluated with the new information of the present measurement.

These operations will be followed in the description of the application of the Kalman filter to the NOMAD-STAR detector.

\subsection{Trajectories of particles in a magnetic field}

The trajectory of a particle inside a constant magnetic field is a helix. Assuming that the magnetic field $B$ (in Tesla) is parallel to the $x$ coordinate, and using the $z$ coordinate as a parameter, then:

$$
\rho \equiv \frac{y^{\prime \prime}[z]}{\left[1+\left(y^{\prime}[z]\right)^{2}\right]^{3 / 2}}=\frac{q B 0.3}{p_{\perp}},
$$

where $\rho=1 / R$ is the curvature, $R$ the radius of curvature (in meters), $q$ the charge of the particle (in units of electron charge) and $p_{\perp}=\sqrt{p_{y}^{2}+p_{z}^{2}}$ the transverse momentum to the magnetic field (in $\mathrm{GeV} / \mathrm{c}$ ) (see Fig. 5). NOMAD-STAR does not have any $x$ information so we do not consider it.



Figure 5: Projected measurements for a Kalman filter track fit.

The solution to this equations is:

$$
y=y_{c}-\tilde{q} \sqrt{R^{2}-\left(z-z_{c}\right)^{2}}
$$


which represents the parametric equation of the y-projection of a helix using the $z$ coordinate as a parameter. The $y z$ projection is a circle (Eq. 4), with $\left(y_{c}, z_{c}\right)$ the center of the circle, $\mathrm{R}$ its radius and $\tilde{q}=q /|q|$ the sign of the charge of the particle. The two possible solutions in Eq. 4 are such that a negatively charged particle, $\tilde{q}=-1$, is bent downwards (in the negative $y$ direction) and a positive one is bent upwards (positive $y$ direction). If $\theta$ is the angle of the track with respect to the $z$ axis in the $y z$ plane (see Fig. 5), the coordinates of the center of the circle are:

$$
\begin{aligned}
& y_{c}=y+\tilde{q} R \cos \theta, \\
& z_{c}=z-\tilde{q} R \sin \theta .
\end{aligned}
$$

The equation of the circle is not linear so, to keep the matrix notation of Eq. 2, we perform a Taylor expansion of Eq. 4 around $z=0$ to linearise the problem:

$$
\begin{gathered}
y=\sum_{n=0}^{\infty} \beta_{n} z^{n}, \\
\beta_{n}=\frac{1}{n !} \frac{d^{n}}{d z^{n}}\left(y_{c}-\tilde{q} \sqrt{R^{2}-\left(z-z_{c}\right)^{2}}\right)_{z=0} .
\end{gathered}
$$

In principle, this expansion implies that the matrices $\mathbf{H}_{k}$ and $\mathbf{x}_{k}$ are of infinite dimension: $\mathbf{H}_{k}=\left\{1, z, z^{2}, z^{3}, \ldots\right\}$ and $\mathbf{x}_{k}=\left\{\beta_{0}, \beta_{1}, \beta_{2}, \ldots\right\}$. However, only three of the $\beta_{n}$ parameters of the expansion are independent because $y$ only depends on $y_{c}, z_{c}$ and $R$. The state vector $\mathbf{x}_{k}$ as defined in Eq. 1 needs to contain only the independent parameters, thus:

$$
y=\left[\begin{array}{l}
1 \\
z \\
z^{2}
\end{array}\right] \cdot\left[\begin{array}{lll}
\beta_{0} & \beta_{1} & \beta_{2}
\end{array}\right]+\sum_{n=3}^{\infty} \beta_{n}\left(\beta_{0}, \beta_{1}, \beta_{2}\right) z^{n}
$$

The number of terms in the expansion to correctly describe the particle trajectories inside NOMAD-STAR is given by the extrapolation errors associated to each term. Fig. 6 shows the extrapolation errors incurred by neglecting $\Delta_{j} \equiv \sum_{n=j}^{\infty} \beta_{n} l_{m a x}^{n}$ in the Taylor expansion, as a function of $p_{\perp}$ and for track angles $10^{\circ}$ and $60^{\circ}$, assuming $B=0.4 \mathrm{~T}$ and that the separation between two consecutive planes is $l_{\max }=3.6 \mathrm{~cm}$. One can see that the cubic term is still needed to ensure that the tracking accuracy remains below the intrinsic resolution of the silicon ladders $(5 \mu \mathrm{m})[8]$ for some tracks with high angles or low momenta. NOMAD-STAR is not sensitive to further terms in the expansion.

The parameters of the equation: $y=a+b z+c z^{2}+d z^{3}\left(+e z^{4}\right)$ are as follows:

$$
\begin{gathered}
a \equiv \beta_{0}=y_{c}-\tilde{q} \sqrt{R^{2}-z_{c}^{2}} \\
b \equiv \beta_{1}=\frac{-\tilde{q} z_{c}}{\sqrt{R^{2}-z_{c}^{2}}}, \\
c \equiv \beta_{2}=\frac{\tilde{q} R^{2}}{2\left(R^{2}-z_{c}^{2}\right)^{3 / 2}}
\end{gathered}
$$



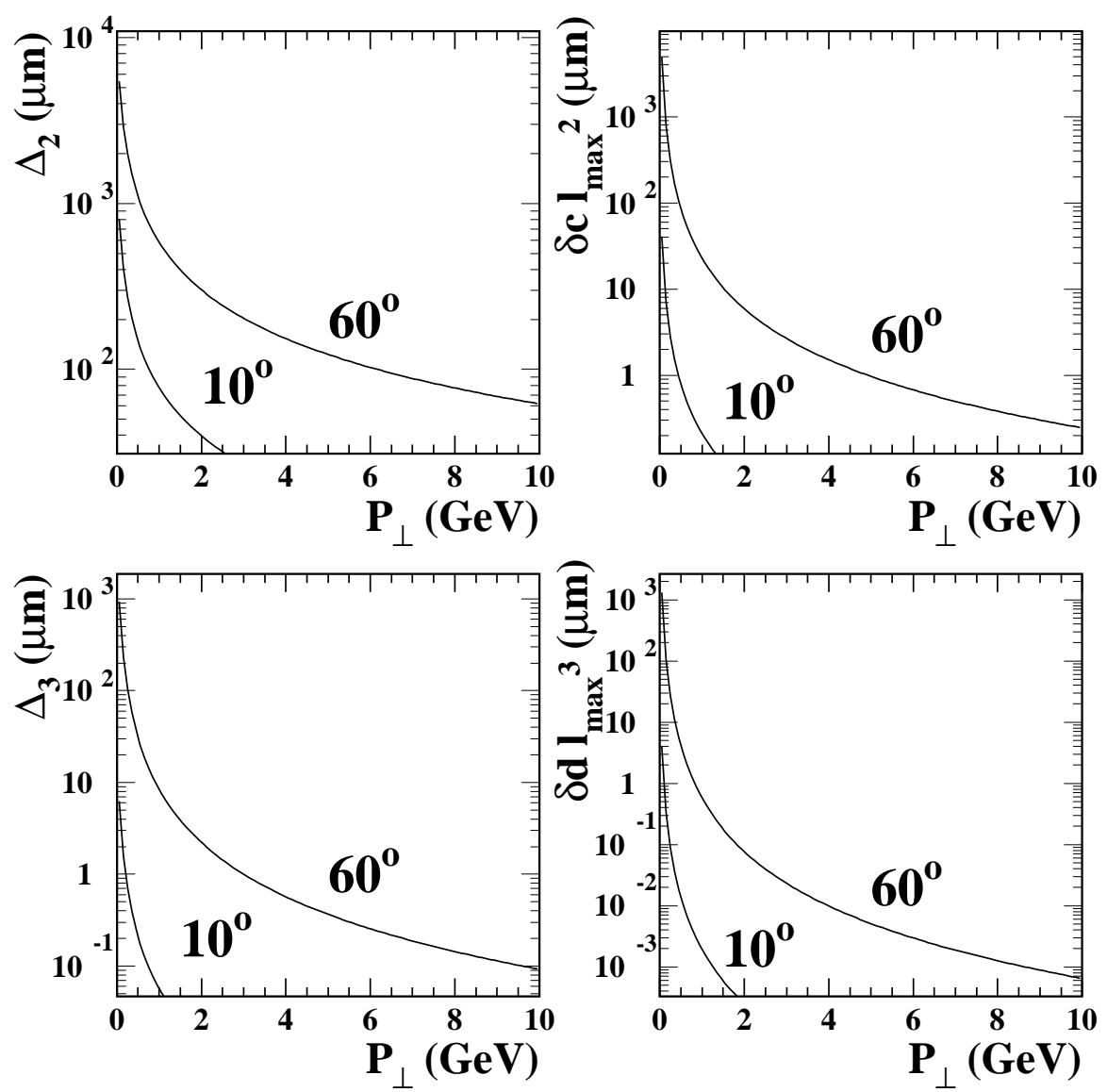

Figure 6: Extrapolation errors incurred by neglecting the terms $\Delta_{2}$ and $\Delta_{3}$ (where $\Delta_{j} \equiv$ $\left.\sum_{n=j}^{\infty} \beta_{n} l_{\text {max }}^{n}\right)$ in the Taylor expansion, assuming the separation between consecutive planes is $3.6 \mathrm{~cm}$, as a function of the momentum $p_{\perp}$ for track angles of $10^{\circ}$ and $60^{\circ}$. Also shown are the uncertainties due to multiple scattering of the quadratic and cubic terms of the expansion: $\delta c l_{\text {max }}^{2}$ and $\delta d l_{\text {max }}^{3}$. 


$$
\begin{gathered}
d \equiv \beta_{3}=\frac{-\tilde{q} R^{2} z_{c}}{2\left(R^{2}-z_{c}^{2}\right)^{5 / 2}}=\frac{2 b c^{2}}{1+b^{2}}, \\
e \equiv \beta_{4}=\frac{\tilde{q} R^{2}\left(R^{2}+4 z_{c}^{2}\right)}{8\left(R^{2}-z_{c}^{2}\right)^{7 / 2}}=\frac{c^{3}\left(1+5 b^{2}\right)}{\left(1+b^{2}\right)^{2}}
\end{gathered}
$$

with $d$ and $e$ dependent on $b$ and $c$. It is also worth noting that, as the Taylor expansion was calculated for the limit $z \rightarrow 0$, it is necessary to change the coordinate system so that the most upstream plane of the measurement defines $z=0$. Now the circle parameters can be written in terms of $a, b$ and $c$ :

$$
\begin{aligned}
& R=\tilde{q} \frac{\left(1+b^{2}\right)^{3 / 2}}{2 c} \\
& y_{c}=a+\frac{\left(1+b^{2}\right)}{2 c} \\
& z_{c}=-\frac{b\left(1+b^{2}\right)}{2 c}
\end{aligned}
$$

\subsection{Kalman track filter}

We now have a simplified scenario where the state vector only has three parameters so we vary the standard implementation of the Kalman Filter to accommodate this circumstance. The measurements $\mathbf{m}_{k}$ are the measured $y_{k}$ positions at a given plane at position $z_{k}$ (see Fig. 5).

The measurement equation (Eq. 2) has to be modified to include the fixed parameter which is not explicitly included in the state vector:

$$
\mathbf{m}_{k}=\mathbf{H}_{k} \mathbf{x}_{k}+d z_{k}^{3}+\epsilon_{k}
$$

The covariance matrix of the measurement:

$$
\operatorname{cov}\left\{\epsilon_{k}\right\}=\mathbf{V}_{k}=\mathbf{G}_{k}^{-1},
$$

is a $1 \times 1$ matrix and is equal to the square of the $y$ resolution of the silicon detectors $\sigma_{y}^{2}$.

The evolution of the state vector is given by Eq. 1. In the absence of energy loss or any other systematic perturbation to the system, this particular choice of state vector should not vary from one plane to another. In that case we have $\mathbf{F}_{k}=\mathbf{I}$, the identity matrix. However in the case of NOMAD-STAR energy loss can be visible for some low momentum particles. The inclusion of energy loss in the Kalman Filter is studied in section 3.3.2.

The process noise, defined in Eq. 1, is included through the covariance matrix of the extrapolation, defined as:

$$
\operatorname{cov}\left\{\omega_{k}\right\}=\mathbf{Q}_{k}
$$


This matrix has two contributions, one related with multiple scattering and the other with energy loss:

$$
\mathbf{Q}_{k}=\mathbf{Q}_{k}^{m s}+\mathbf{Q}_{k}^{e l}
$$

which are studied in sections 3.3.1 and 3.3.2, respectively.

\subsubsection{Multiple Coulomb scattering}

Multiple scattering was added to the Kalman filter for a parabolic track model in Ref. [22]. In this section we will generalize this for a cubic model with a more accurate multiple scattering algorithm.

The multiple scattering theory of Molière [23], reformulated by Bethe [24], can be parametrised by a Gaussian approximation [25], where the width of the distribution is given by:

$$
\theta_{m s}^{2}=\frac{\chi_{c}^{2}}{1+F^{2}}\left[\frac{1+v}{v} \ln (1+v)-1\right],
$$

with:

$$
v=\frac{0.5 \Omega}{1-F}
$$

and where:

$$
\Omega=b_{c} q^{2} \frac{s}{\beta^{2}}
$$

is the mean number of scatters, $s$ is the path length of the particle and $F=0.98$ is the fraction of tracks considered in the Gaussian distribution. The critical scattering angle [26] is:

$$
\chi_{c}=\chi_{c c} q \frac{\sqrt{s}}{p_{\perp} \beta}
$$

with $\chi_{c c} \approx\left(0.39612 \times 10^{-3}\right)^{2} Z_{s}^{\prime} \frac{\rho}{W}, q$ and $\beta$ the charge and speed of the incident particle, $\rho$ and $W$ the density and molecular weight of the material, and:

$$
b_{c} \approx 6702.33 \rho Z_{s}^{\prime} e^{\left(Z_{x}^{\prime}-Z_{E}^{\prime}\right) / Z_{s}^{\prime}} .
$$

For a mixture or compound, the following variables are defined:

$$
\begin{gathered}
Z_{s}^{\prime}=\sum_{i=1}^{N} \frac{p_{i}}{A_{i}} Z_{i}\left(Z_{i}+1\right), \\
Z_{E}^{\prime}=\sum_{i=1}^{N} \frac{p_{i}}{A_{i}} Z_{i}\left(Z_{i}+1\right) \ln \left(Z_{i}^{-2 / 3}\right), \\
Z_{x}^{\prime}=\sum_{i=1}^{N} \frac{p_{i}}{A_{i}} Z_{i}\left(Z_{i}+1\right) \ln \left[1+3.34\left(\frac{q Z_{i}}{137 \beta}\right)^{2}\right],
\end{gathered}
$$


where $Z_{i}$ and $A_{i}$ are the atomic number and atomic weight of each element in the mixture, $p_{i}$ is the proportion by weight of that element and $N$ the total number of elements. This approximation reproduces the Molière theory with an accuracy of $2 \%$.

For an incident particle of $q= \pm 1$, and for the case of boron carbide, the term $\frac{1+v}{v} \approx 1$ and then Eq. 22 becomes:

$$
\theta_{m s}^{2}=k s[\ln (1+B s)-1],
$$

with $k=5.8335 \times 10^{-7} /\left(p_{\perp}^{2} \beta^{2}\right)$ and $B=\frac{402685}{\beta^{2}} e^{-4.944 \times 10^{-3} / \beta^{2}}$ (if $s$ is in $\mathrm{cm}$ and $p_{\perp}$ is in $\mathrm{GeV} / \mathrm{c})$.

We can assume that, locally, the particle trajectory is a straight line. In that case

$$
\delta\left(\theta_{m s}^{2}\right)=k \delta s\left[\ln (1+B s)-\frac{1}{1+B s}\right]=k L(z) G(z) \delta z
$$

where:

$$
\begin{gathered}
L(z)=1 / \cos \theta=\sqrt{1+\left[y^{\prime}(z)\right]^{2}}, \\
s(z)=\int_{z_{i}}^{z} L\left(z^{\prime}\right) d z^{\prime}, \\
G(z)=\ln (1+B s(z))-\frac{1}{1+B s(z)},
\end{gathered}
$$

with $z_{i}, z_{f}$ the limits of each of the $B_{4} C$ planes. The integral of $s(z)$ can be calculated analytically:

$$
s(z)=R\left[\arcsin \left(\tilde{q} \frac{z-z_{c}}{R}\right)-\arcsin \left(\tilde{q} \frac{z_{i}-z_{c}}{R}\right)\right]
$$

and using Eq. 15 and 17

$$
s(z)=R\left[\arcsin \left(\frac{2 c z+b\left(1+b^{2}\right)}{\left(1+b^{2}\right)^{3 / 2}}\right)-\arcsin \left(\frac{2 c z_{i}+b\left(1+b^{2}\right)}{\left(1+b^{2}\right)^{3 / 2}}\right)\right] .
$$

Explicitly, the fitting parameters $\beta_{n}$ of the track model depend on the multiple scattering angle. The multiple scattering contribution to the covariance matrix of the prediction, $Q_{k}^{m s}$, is a $3 \times 3$ matrix with terms:

$$
\left(\mathbf{Q}_{k}^{m s}\right)_{i j}=\operatorname{cov}_{\theta}\left\{\beta_{i}, \beta_{j}\right\}=\int \frac{\delta \beta_{i}}{\delta \theta} \frac{\delta \beta_{j}}{\delta \theta} \delta\left(\theta^{2}\right)=k \int_{z_{i}}^{z_{f}} \frac{\delta \beta_{i}}{\delta \theta} \frac{\delta \beta_{j}}{\delta \theta} L(z) G(z) \delta z
$$

where the integration is needed for non-straight particles. To calculate the terms of this matrix we use the relation:

$$
\delta \beta_{n}=-(n+1) \beta_{n+1} \delta z_{c}, n>0,
$$

which is a consequence of Eq 8. We also use the continuity of $y(z)$ :

$$
0=\delta y=\delta a+\sum_{n=1}^{\infty} \delta \beta_{n} z^{n}=\delta a-\delta z_{c} \sum_{n=1}^{\infty}(n+1) \beta_{n+1} z^{n}=\delta a-\left(y^{\prime}-b\right) \delta z_{c}
$$


and the relation between the multiple scattering angle and $y^{\prime}(z)=\tan \theta$ :

$$
\left(1+y^{\prime 2}\right) \delta \theta=\delta y^{\prime}=-y^{\prime \prime} \delta z_{c}
$$

This last equation implies:

$$
\delta z_{c}=\frac{-\left(1+y^{\prime 2}\right)}{y^{\prime \prime}} \delta \theta=\frac{-L(z)^{2}}{y^{\prime \prime}} \delta \theta .
$$

Using equations 38,39 and 41 it is straight-forward to obtain the desired quantities:

$$
\begin{gathered}
\frac{\delta a}{\delta \theta}=-\left(y^{\prime}-b\right) \frac{L(z)^{2}}{y^{\prime \prime}}, \\
\frac{\delta \beta_{n}}{\delta \theta}=(n+1) \beta_{n+1} \frac{L(z)^{2}}{y^{\prime \prime}}, n>0 .
\end{gathered}
$$

Defining the following integrals:

$$
\begin{gathered}
I_{1}=\int_{z_{i}}^{z_{f}} \frac{\left(y^{\prime}(z)-b\right)^{2} L(z)^{5}}{\left[y^{\prime \prime}(z)\right]^{2}} G(z) d z, \\
I_{2}=\int_{z_{i}}^{z_{f}} \frac{\left(y^{\prime}(z)-b\right) L(z)^{5}}{\left[y^{\prime \prime}(z)\right]^{2}} G(z) d z, \\
I_{3}=\int_{z_{i}}^{z_{f}} \frac{L(z)^{5}}{\left[y^{\prime \prime}(z)\right]^{2}} G(z) d z,
\end{gathered}
$$

the covariance matrix can now be written:

$$
\mathbf{Q}_{k}^{m s}=\left[\begin{array}{ccc}
k I_{1} & -2 c k I_{2} & -3 d k I_{2} \\
-2 c k I_{2} & 4 c^{2} k I_{3} & 6 c d k I_{3} \\
-3 d k I_{2} & 6 c d k I_{3} & 9 d^{2} k I_{3}
\end{array}\right]
$$

In the case of the cubic equation: $y^{\prime}(z)=b+2 c z+3 d z^{2}$ and $y^{\prime \prime}(z)=2 c+6 d z$. These integrals are performed numerically for each $\mathrm{B}_{4} \mathrm{C}$ plane traversed. The error terms $\delta c=$ $\sqrt{9 d^{2} k I_{3}}$ and $\delta d=\sqrt{16 e^{2} k I_{3}}$ are shown in Fig. $6 . \delta d$ is found to be negligible for the case of NOMAD-STAR.

\subsubsection{Energy loss}

The trajectory of particles in dense media is affected by energy loss. For moderately relativistic particles other than electrons, the mean rate of energy loss is given by the well known Bethe-Bloch formula [27].

As is shown in Eq. 3, the radius of the circle is proportional to $p_{\perp}$. As the particle loses energy, the radius of the circle does not remain constant and the trajectory is like a spiral. To simplify the Kalman filter formalism we can assume that, locally, the trajectory of the particle is still a circle (Fig.7). Energy loss changes the parameters of the circle in a continuous way from one point to the next. That implies the continuity of $y(z)$ and $y^{\prime}(z)$. The infinitesimal variation in the circle parameters can be written in terms of $\delta R, \delta y_{c}$ and 




Figure 7: Variation of the circle parameters induced by energy loss.

$\delta z_{c}$, but only one of these variations is independent because of the two constrains $\delta y(z)=0$ and $\delta y^{\prime}(z)=0$. Starting from equation 4 , it is easy to obtain:

$$
\begin{aligned}
& \delta y_{c}=-\left(y-y_{c}\right) \frac{\delta R}{R}, \\
& \delta z_{c}=-\left(z-z_{c}\right) \frac{\delta R}{R},
\end{aligned}
$$

which can also be intuitively extracted from figure 7 . The variation in the expansion coefficients is:

$$
\delta \beta_{n}=\frac{\partial \beta_{n}}{\partial R} \delta R+\frac{\partial \beta_{n}}{\partial y_{c}} \delta y_{c}+\frac{\partial \beta_{n}}{\partial z_{c}} \delta z_{c} .
$$

Introducing the derivatives of equations 10, 11 and 12 into 50, and using also 48 and 49, we obtain:

$$
\begin{gathered}
\delta a=-\frac{\delta R}{R} \sum_{n=2}^{\infty} \beta_{n} z^{n} \simeq-c z^{2} \frac{\delta R}{R}, \\
\delta b=2 c z \frac{\delta R}{R} \\
\delta c=(-c+3 d z) \frac{\delta R}{R} \simeq-c \frac{\delta R}{R} .
\end{gathered}
$$



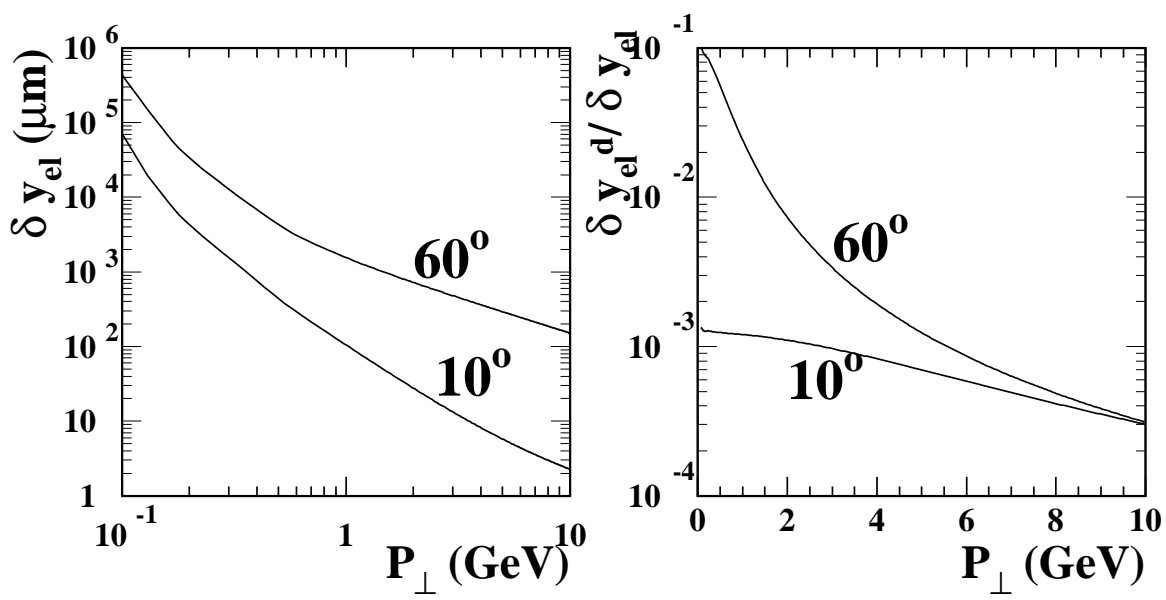

Figure 8: a) Extrapolation error incurred by neglecting the energy loss effect assuming a single $B_{4} C$ layer and a separation between consecutive planes of $3.6 \mathrm{~cm}$, (only the dominant terms up to $c$ included) as a function of the momentum for different angles. b) Ratio between the $d$-term and the dominant terms up to $c$ of the extrapolation error due to energy loss.

These two approximations are needed if we want to keep the formalism of Eq. 1. Figure $8 \mathrm{~b}$ ) shows the ratio between the terms containing $d$ and the dominant terms up to the one containing $c$ of equations 51, 52 and 53. In general, this ratio is very small, thereby justifying the previous approximations. Now we have to find out how the radius of the circle is affected by energy loss:

$$
\frac{d R}{R}=\frac{d p_{\perp}}{p_{\perp}}=\frac{d E}{E}=\frac{\beta}{p} \frac{d E}{d s} d s .
$$

At a given $\mathrm{z}$, the momentum of the particle can be calculated by integrating the BetheBloch formula for energy loss along the length of $B_{4} C$ :

$$
p(z)=\int \beta \frac{d E}{d s} d s=\int_{z_{i}}^{z_{f}} \beta \frac{d E}{d s} \frac{L(z)}{\cos \gamma} d z,
$$

where $\gamma$ is the angle between $\mathbf{p}$ and $\mathbf{p}_{\perp}$ and $\beta=p / E$. This integral can be performed numerically, but if one assumes that the energy loss can be approximated by a power law:

$$
\frac{d E}{d s}=\alpha_{1} p^{\alpha_{2}}
$$

then the integral can be performed analytically (assuming $\beta$ constant):

$$
p(z)=\left[p_{i}^{1-\alpha_{2}}+\beta\left(1-\alpha_{2}\right) \alpha_{1} s(z)\right]^{1 /\left(1-\alpha_{2}\right)},
$$




\begin{tabular}{ccc}
\hline \hline Momentum range $(\mathrm{GeV} / \mathrm{c})$ & $\alpha_{1}$ & $\alpha_{2}$ \\
\hline$p_{\perp}<0.17$ & $5.729 \times 10^{-4}$ & -1.321 \\
$0.17<p_{\perp}<0.51$ & $3.715 \times 10^{-3}$ & -0.2636 \\
$p_{\perp}>0.51$ & $4.615 \times 10^{-3}$ & 0.06141 \\
\hline \hline
\end{tabular}

Table 1: Parameters for power law parametrizations (Eq. 56) of the Bethe-Bloch energy loss formula for $B_{4} C$. With these parameters, the energy loss is in units of $\mathrm{GeV} \mathrm{cm}^{-1}$.

with $p_{i}$ the momentum at the initial plane. Using equations 54,56 :

$$
\frac{d R}{R}=\frac{\beta \alpha_{1}}{p_{i}^{1-\alpha_{2}} \cos \gamma} \frac{1}{1+\frac{\beta\left(1-\alpha_{2}\right) \alpha_{1} s(z)}{p_{i}^{1-\alpha_{2}}}} L(z) d z,
$$

We now combine Eqs. 51, 52, 53 and 58, and integrate over the thickness of the $B_{4} C$ plates, to obtain the $\mathbf{F}_{\mathbf{k}}$ matrix of Eq. 1:

$$
\mathbf{F}_{k}=\left[\begin{array}{ccc}
1 & 0 & -k_{e l} \int_{z_{i}}^{z_{f}} z^{2} F(z) L(z) d z \\
0 & 1 & 2 k_{e l} \int_{z_{f}}^{z_{f}} z F(z) L(z) d z \\
0 & 0 & 1-k_{e l} \int_{z_{i}}^{z_{f}} F(z) L(z) d z
\end{array}\right]
$$

where the parameter $k_{e l}$ is:

$$
k_{e l}=\frac{\beta \alpha_{1}}{p_{i}^{1-\alpha_{2}} \cos \gamma}
$$

and $F(z)$ is defined as:

$$
F(z)=\frac{1}{1+\frac{\beta\left(1-\alpha_{2}\right) \alpha_{1} s(z)}{p_{i}^{1-\alpha_{2}}}} .
$$

Notice that at first order, the variation of the state vector between two silicon planes induced by energy loss depends only on the quadratic parameter $c$ (equations 51, 52 and $53)$.

We have parametrized the Bethe-Bloch formula for $B_{4} C$ (with $d E / d s$ in units of $\mathrm{GeV}$ $\mathrm{cm}^{-1}$ ) using Eq. 56, with the parameters given in table 1 for relevant momentum ranges. This approximation is accurate to better than $10 \%$ below $1 \mathrm{GeV} / \mathrm{c}$, and is better than $0.5 \%$ between $1 \mathrm{GeV} / \mathrm{c}$ and $10 \mathrm{GeV} / \mathrm{c}$. Figure 8 a) shows the extrapolation error incurred if we do not take into account energy loss in the Kalman filter matrix (Eq. 59).

Eq. 56 gives the mean rate of energy loss, but in fact fluctuations in energy loss follow a Landau distribution, which is approximately Gaussian for thick media. These random fluctuations contribute to the process noise and are included in the Kalman filter mechanism through the covariance matrix $\mathbf{Q}_{k}^{e l}$ defined in Eq. 21. In NOMAD-STAR, the energy loss effect is small, so the error induced by this effect is even smaller and it can be neglected when we add it in quadrature (see eq. 21) to the multiple scattering error. 
Having determined the track model and process noise, the Kalman track filter can now be broken down into its three constituent phases: prediction, filter and smoother (see for example Ref. [11]).

\subsubsection{Prediction}

A prediction of the state vector $\mathbf{x}_{k}^{P}$ made at measurement plane $k$ is based on the state vector information at plane $k-1$.

The NOMAD drift chambers provide tracking and momentum information for each of the reconstructed tracks. The initial conditions for the state vector are given by the first silicon hit position, which defines $a$, and the parameters $b, c$ and $d$ (Eqs. 11-13) as given by the drift chambers.

Given the covariance matrix of the state vector as:

$$
\mathbf{C}_{k}=\operatorname{cov}\left\{\mathbf{x}_{k}^{P}-\mathbf{x}_{k}\right\}
$$

the extrapolation of the covariance matrix (the prediction for this covariance matrix given the knowledge of this matrix from previous steps) is:

$$
\mathbf{C}_{k}^{P}=\mathbf{F}_{k} \mathbf{C}_{k-1} \mathbf{F}_{k}^{T}+\mathbf{Q}_{k} .
$$

Again, the initial conditions for the covariance matrix are given by the resolution of the first silicon hit and the errors in the parameters as determined by the drift chamber fit.

The residuals of the prediction from the measurement at plane $k$ is:

$$
\mathbf{r}_{k}^{P}=\mathbf{m}_{k}-\mathbf{H}_{k} \mathbf{x}_{k}^{P}-d z_{k}^{3} .
$$

The covariance of this residual is then:

$$
\mathbf{R}_{k}^{P}=\mathbf{V}_{k}+\mathbf{H}_{k} \mathbf{C}_{k}^{P} \mathbf{H}_{k}^{T}+z_{k}^{6} \sigma_{d d}^{2},
$$

where $\sigma_{d d}^{2}$ is the square of the error in the parameter $d$ due to the uncertainty in the measured track momentum.

\subsubsection{Filter}

The filtering process now incorporates information from the measurement at plane $k$ into the state vector $\mathbf{x}_{k}$ :

$$
\mathbf{x}_{k}=\mathbf{x}_{k}^{P}+\mathbf{K}_{k} \mathbf{r}_{k}^{P},
$$

where $\mathbf{K}_{k}$ is known as the Kalman gain matrix that updates the relationship between the state vector and the measurements by including the new information:

$$
\mathbf{K}_{k}=\mathbf{C}_{k}^{P} \mathbf{H}_{k}^{T} \mathbf{R}_{k}^{-1} .
$$

The updated covariance matrix is then: 


$$
\mathbf{C}_{k}=\left(\mathbf{I}-\mathbf{K}_{k} \mathbf{H}_{k}\right) \mathbf{C}_{k}^{P}
$$

with the filtered residuals:

$$
\mathbf{r}_{k}=\mathbf{m}_{k}-\mathbf{H}_{k} \mathbf{x}_{k}-d z_{k}^{3}
$$

and the covariance matrix of the filtered residuals:

$$
\mathbf{R}_{k}=\left(\mathbf{I}-\mathbf{H}_{k} \mathbf{K}_{k}\right) \mathbf{V}_{k}+z_{k}^{6} \sigma_{d d}^{2} .
$$

The $\chi^{2}$ of measurement $k$ at this stage of the filtering process is:

$$
\chi_{k}^{2}=\mathbf{r}_{k}^{T} \mathbf{R}_{k}^{-1} \mathbf{r}_{k}
$$

\subsubsection{Smoother}

Once every measurement has been filtered, the smoother is then used to propagate all the information added during the filtering process to a given measurement plane. The superscript $S$ is used to denote the value after the smoothing operation. The smoothed state vector is:

$$
\mathbf{x}_{k}^{S}=\mathbf{x}_{k}+\mathbf{A}_{k}\left(\mathbf{x}_{k+1}^{S}-\mathbf{x}_{k+1}^{P}\right)
$$

with:

$$
\mathbf{A}_{k}=\mathbf{C}_{k} \mathbf{F}_{k+1}^{T}\left(\mathbf{C}_{k+1}^{P}\right)^{-1}
$$

and the smoothed covariance matrix, residuals and covariance of the residuals being:

$$
\begin{gathered}
\mathbf{C}_{k}^{S}=\mathbf{C}_{k}+\mathbf{A}_{k}\left(\mathbf{C}_{k+1}^{S}-\mathbf{C}_{k+1}^{P}\right) \mathbf{A}_{k}^{T}, \\
\mathbf{r}_{k}^{S}=\mathbf{m}_{k}-\mathbf{H}_{k} \mathbf{x}_{k}^{S}-d z_{k}^{3}, \\
\mathbf{R}_{k}^{S}=\mathbf{V}_{k}-\mathbf{H}_{k} \mathbf{C}_{k}^{S} \mathbf{H}_{k}^{T}+z_{k}^{6} \sigma_{d d}^{2} .
\end{gathered}
$$

The $\chi^{2}$ also has to be modified for the smoothed values:

$$
\chi_{k}^{2^{S}}=\left(\mathbf{r}_{k}^{S}\right)^{T}\left(\mathbf{R}_{k}^{S}\right)^{-1} \mathbf{r}_{k}^{S} .
$$

The three step process of prediction, filtering and smoothing is iterated for all the measurement planes up to and including the information from the most upstream plane. The whole procedure then gives the track parameters at the plane closest to the interaction point. 




Figure 9: Schematic of the Kalman vertex fit.

\subsection{Kalman vertex fit}

Once the track fit has been achieved, the vertex fit can commence. The vertex fit is also an iterative procedure and is similar to the track fit, except that the measurements now consist of the track parameters determined in the track fit, $\mathbf{P}_{k}=\{a, b, c, d\}$, and the state vector becomes the position of the vertex $\mathbf{x}=\{y, z\}$ (see Fig 9). Each track is weighted according to the inverse of the covariance matrix of the measurements:

$$
\mathbf{G}_{k}=\left(\operatorname{cov}\left(\mathbf{P}_{k}\right)\right)^{-1}
$$

The tracks with lower momentum will have lower weights, due to the effects of multiple scattering, and so will have a smaller effect on the vertex position. These low momentum tracks have the largest values of the parameters $c$ and $d$ and so in order to save computing time without any significant penalty in vertex accuracy, a new set of track parameters is used: $\mathbf{P}_{k}=\left\{a, b, c^{\prime}\right\}$, where the parameters $a$ and $b$ are the same as before, but the new parameter $c^{\prime}=c+d \cdot z$, with $z$ the coordinate of the vertex after the last filter (or the initial estimate, if this is the first iteration). This produces a local approximation to the cubic track model which is accurate as long as the vertex position does not move significantly during the filtering stage.

The covariance matrix of the state vector will again be represented by $\mathbf{C}_{k}$. An additional vector $\mathbf{Q}_{k}=\left\{b, c^{\prime}\right\}$ is introduced to represent the angle and magnitude of the momentum of track $k$ at the vertex. The measurement equation contains the track model: 


$$
\mathbf{P}_{k}=\mathbf{H}_{k}\left(\mathbf{x}_{k}, \mathbf{Q}_{k}\right)+\epsilon_{k}
$$

with the function $\mathbf{H}$ defined as:

$$
\begin{gathered}
\mathbf{H}(0)=y-b z-c^{\prime} z^{2}, \\
\mathbf{H}(1)=\frac{y-a}{z}-c^{\prime} z \\
\mathbf{H}(2)=\frac{y-a}{z^{2}}-\frac{b}{z} .
\end{gathered}
$$

In order to complete the vertex fit, it is necessary to introduce 2 additional matrices:

$$
\begin{gathered}
\mathbf{A}_{k}=\frac{\delta \mathbf{P}_{k}}{\delta \mathbf{x}_{k}}=\left[\begin{array}{cc}
1 & -b-2 c^{\prime} z \\
\frac{1}{z} & \frac{a-y}{z^{2}}-c^{\prime} \\
\frac{1}{z^{2}} & \frac{2(a-y)}{z^{3}}+\frac{b}{z^{2}}
\end{array}\right], \\
\mathbf{B}_{k}=\frac{\delta \mathbf{P}_{k}}{\delta \mathbf{Q}_{k}}=\left[\begin{array}{cc}
0 & 0 \\
1 & 0 \\
0 & 1
\end{array}\right],
\end{gathered}
$$

such that the function $\mathbf{H}_{k}$ is linear around the point $\left(\mathbf{x}_{k, 0}, \mathbf{Q}_{k, 0}\right)$ :

$$
\mathbf{H}_{k}\left(\mathbf{x}_{k, 0}, \mathbf{Q}_{k, 0}\right)=\mathbf{c}_{k, 0}+\mathbf{A}_{k} \mathbf{x}_{k, 0}+\mathbf{B}_{k} \mathbf{Q}_{k, 0}
$$

and serves as a definition for $\mathbf{c}_{k, 0}$. We can then proceed to perform the stages of the Kalman vertex filter as outlined in [11].

\subsubsection{Prediction}

The prediction equations are approximated by the parameters at the last measurement plane:

$$
\begin{gathered}
\mathbf{x}_{k}^{P}=\mathbf{x}_{k-1}, \\
\mathbf{C}_{k}^{P}=\mathbf{C}_{k-1} .
\end{gathered}
$$

\subsubsection{Filter}

The new state vector after filtering is:

$$
\mathbf{x}_{k}=\mathbf{C}_{k}\left[\left(\mathbf{C}_{k-1}\right)^{-1} \mathbf{x}_{k-1}+\mathbf{A}_{k}^{T} \mathbf{G}_{k}^{B}\left(\mathbf{P}_{k}-\mathbf{c}_{k, 0}\right)\right],
$$

where the following matrices are also defined:

$$
\mathbf{C}_{k}=\left[\left(\mathbf{C}_{k-1}\right)^{-1}+\mathbf{A}_{k}^{T} \mathbf{G}_{k}^{B} \mathbf{A}_{k}\right]^{-1},
$$




$$
\begin{gathered}
\mathbf{G}_{k}^{B}=\mathbf{G}_{k}-\mathbf{G}_{k} \mathbf{B}_{k} \mathbf{W}_{k} \mathbf{B}_{k}^{T} \mathbf{G}_{k}, \\
\mathbf{W}_{k}=\left[\mathbf{B}_{k}^{T} \mathbf{G}_{k} \mathbf{B}_{k}\right]^{-1} .
\end{gathered}
$$

The $\chi^{2}$ of each filter step has 2 degrees of freedom:

$$
\begin{aligned}
\chi_{k, F}^{2}= & \left(\mathbf{P}_{k}-\mathbf{c}_{k, 0}-\mathbf{A}_{k} \mathbf{x}_{k}-\mathbf{B}_{k} \mathbf{Q}_{k}\right)^{T} \mathbf{G}_{k}\left(\mathbf{P}_{k}-\mathbf{c}_{k, 0}-\mathbf{A}_{k} \mathbf{x}_{k}-\mathbf{B}_{k} \mathbf{Q}_{k}\right)+ \\
& \left(\mathbf{x}_{k}-\mathbf{x}_{k-1}\right)^{T}\left(\mathbf{C}_{k-1}\right)^{-1}\left(\mathbf{x}_{k}-\mathbf{x}_{k-1}\right),
\end{aligned}
$$

so the total $\chi^{2}$ of the fit after adding $k$ tracks is just:

$$
\chi_{k}^{2}=\chi_{k-1}^{2}+\chi_{k, F}^{2}
$$

The filter is recomputed until there is no significant change in the $\chi^{2}$ or in the parameter estimates.

\subsubsection{Inverse filter}

It is also possible to remove a track from a vertex fit by applying the inverse filter. The procedure is identical to the filter except in the sign of the matrix $A_{k}$ :

$$
\mathbf{x}_{k}=\mathbf{C}_{k}\left[\left(\mathbf{C}_{k-1}\right)^{-1} \mathbf{x}_{k-1}-\mathbf{A}_{k}^{T} \mathbf{G}_{k}^{B}\left(\mathbf{P}_{k}-\mathbf{c}_{k, 0}\right)\right]
$$

with:

$$
\mathbf{C}_{k}=\left[\left(\mathbf{C}_{k-1}\right)^{-1}-\mathbf{A}_{k}^{T} \mathbf{G}_{k}^{B} \mathbf{A}_{k}\right]^{-1}
$$

\subsubsection{Smoother}

The smoother does not make any changes to the vertex position since it is assumed that there is no process noise. Rather, it finds the parameters of each track at the final vertex position:

$$
\begin{gathered}
\mathbf{x}_{k}^{S}=\mathbf{x}_{k}, \\
\mathbf{C}_{k}^{S}=\mathbf{C}_{k}, \\
\mathbf{Q}_{k}^{S}=\mathbf{W}_{k} \mathbf{B}_{k}^{T} \mathbf{G}_{k}\left(\mathbf{P}_{k}-\mathbf{c}_{k, 0}-\mathbf{A}_{k} \mathbf{x}_{k}\right) .
\end{gathered}
$$




\subsubsection{Initial conditions}

It has been found that the result of the vertex fit is very sensitive to the initial conditions that are passed to it. This is a peculiarity of a fixed target neutrino experiment, where there is no a priori vertex estimate, as opposed to the case of collider experiments or to other fixed target experiments where a well defined target region is defined. An initial estimate for the vertex position and the covariance matrix for this estimate need to be chosen with some care. If the initial vertex position is chosen to be at the origin (or at some other arbitrary location) and the covariance matrix correspondingly large, the fit may not converge quickly (or not at all) as the majority of events in NOMAD-STAR have low multiplicity. If the initial covariance matrix is too small then no matter where the initial position of the vertex is chosen, the filter will have a very small effect compared to the weight of the initial estimate of the track parameters. As a result, the initial estimate should have some physical basis and, in our case, it is made by finding the crossing point of at least 2 tracks while determining the accuracy of this initial estimate by studying Monte Carlo events.

An additional consideration is raised when there are three or more tracks in the vertex fit. A typical $\nu_{\mu}$ charged current interaction with three or more tracks will contain a $\mu^{-}$ with a large momentum and several other hadronic tracks of lower momenta. It would be tempting to take the $\mu^{-}$and the highest momentum hadronic track to calculate the initial vertex estimate, as this combination suffers least from multiple scattering. This can cause a problem, however, as the initial vertex will lie exactly on the extrapolated paths of the two highest momenta (and thus highest weight) tracks and the filter will fail to effectively incorporate information from the other lower momentum tracks. In practice, this effect causes the vertex position to only move up and down the path of the highest momentum track. The solution is to take at least three tracks and find the centre of the triangle defined by the 3 crossing points of these tracks. The filter will see that the vertex position does not agree perfectly with any given track and will thus be free to move the vertex to accommodate all the tracks in the fit, weighted appropriately.

\section{The NOMAD-STAR alignment}

The alignment of the NOMAD-STAR detector with muons traversing it [28] serves as an example of the use of the Kalman filter for tracking purposes. The alignment procedure relies heavily on the Kalman filter to produce the best estimate for the track parameters that minimise the relative positions of each of the silicon detectors in NOMAD-STAR. The alignment of the detector is also crucial in order to achieve good spatial resolution.

The signals of a given strip within a ladder only give us the relative position of hits with respect to the ladder, but not its position in the global reference system. Even within a ladder, it is important to know the relative positions of each of the 12 detectors. Although the detectors were glued nearly parallel to each other, the accuracy of this gluing is not sufficient to determine the strip coordinate along the $72 \mathrm{~cm}$ length of a ladder. Instead, a strip inside a ladder defines a series of segments, corresponding to each bonded detector, rather than a straight line (Fig. 10). 


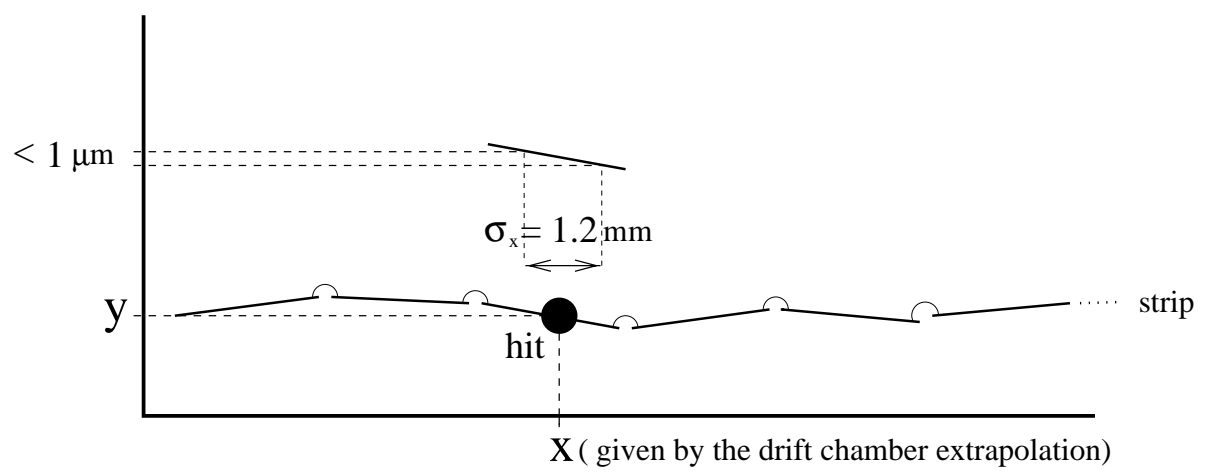

Figure 10: Strip along a ladder.

Thus, to know the location of the hit in the global system, the $x$ coordinate of the hit is needed as well as the exact position of each detector in the global reference frame. The $x$ position of the hit is given by extrapolating back from the NOMAD drift chambers (DC). As the strips inside a ladder are nearly parallel to the $x$ axis, the error in the $y$ position of a hit coming from the error in $x$ is negligible, so the DC resolution $\Delta x_{D C} \sim 1.2 \mathrm{~mm}[2]$ is sufficient.

\subsection{Optical survey}

An optical survey of all the detector positions before the installation of NOMAD-STAR inside the NOMAD detector was performed to serve as a seed for the more general alignment using muons. The optical survey was done in the laboratory and has been described elsewhere [9, 29].

The surveying was performed using a CCD camera with magnifying optics, mounted on a measuring table. The camera could be moved independently along the three axial direction in increments of $1 \mu \mathrm{m}$ with the aid of stepping-motors. The positions of four points per detector for all the ladders and layers of NOMAD-STAR were measured with the surveying table. The survey was performed initially for each of the isolated frames of NOMAD-STAR (consisting of 10 ladders of 12 detectors each ladder). The systematic errors associated to the survey of the individual layers in the $x, y$ and $z$ positions were determined to be $6.4 \mu \mathrm{m}$, $6.6 \mu \mathrm{m}$ and $14.1 \mu \mathrm{m}$, respectively. Then, the layers were installed inside the support basket (also called the mini-basket). Due to constraints in the measuring system, only the central detectors for each layer were able to be surveyed once they were installed inside the minibasket. For these detectors, the added constraint of the support frame modified their $z$ position and degraded their resolution $(31 \mu \mathrm{m})$ but kept the $x$ and $y$ positions unaltered. 


\subsection{Alignment seeds}

Once the optical survey was performed, the NOMAD-STAR mini-basket was installed in NOMAD. The coordinates of the silicon detectors inside the NOMAD frame were determined by external survey measurements with a precision of $0.4 \mathrm{~mm}$. The positions of all the detectors were then transformed into the general NOMAD frame but with this much coarser resolution.

The position of an individual detector in space is defined by one translation $\vec{r}_{0}$ and one rotation $\mathbf{R}$, which can be described by three angles or three orthogonal unitary vectors $(\vec{u}, \vec{v}, \vec{w})$. Via a $\chi^{2}$ minimization with a planar model, the four points per detector from the survey are transformed into the quantities $\vec{r}_{0}, \vec{u}, \vec{v}, \vec{w}$. Using this constraint, the information is optimized and the survey errors are reduced (from $\sigma_{y_{0}}=6.6 \mu \mathrm{m}$ to $4.0 \mu \mathrm{m}$ ). The optical survey gives the initial $\vec{r}_{0}, \vec{u}, \vec{v}, \vec{w}$ which serves as the starting point for the alignment of the detectors with muons.

Although the relative $y$ position resolution of each individual detector was around $4 \mu \mathrm{m}$, possible internal movements in NOMAD-STAR during and after installation and the coarse resolution of the external survey means that we have poor knowledge of the absolute positioning of each element inside the global NOMAD reference frame. It is assumed that movements of the detectors inside a ladder are negligible, so it is only necessary to perform a ladder-by-ladder alignment. The position of a ladder is defined by the position of the first detector. The relative positions of individual detectors inside a ladder are then given by the results of the survey. Allowances are made for rotations and shifts of the ladders within each of the planes, which are nearly parallel to the $x y$ plane. In addition, due to the mechanical freedom of each frame inside the mini-basket, allowance has to be made for rotations and shifts involving the $z$ coordinate of each frame.

\subsection{Muon selection}

The alignment was performed by using energetic muons passing through the detector. These muons are those from the flat-top of the SPS beam as selected by special triggers in NOMAD [30].

The resolution in $z$ becomes smaller as the angle of the muon increases. Since the muons available for the alignment are mostly perpendicular to the silicon planes, the information about the $z$ position of the detectors is minimal. In Fig. 11 it is possible to see a correlation between the angular distribution of the muons with their momentum (the average angle being larger for low energy muons).

Two independent alignments were performed to optimize the $x y$ projections of the detectors and the projection involving the $z$ coordinate:

- Alignment in xy: High momentum muons with a very small angle $(p>50 \mathrm{GeV}$, $\left.\theta_{\text {muon }} \in[-0.5,0.5]^{\circ}\right)$. If the angle is small the alignment in $x y$ does not depend on the $z$ position of the ladders.

- Alignment in z: High angle muons $\left(\theta_{\text {muon }} \in[-10,-2.5]^{\circ}\right)$, which imply low momentum. 


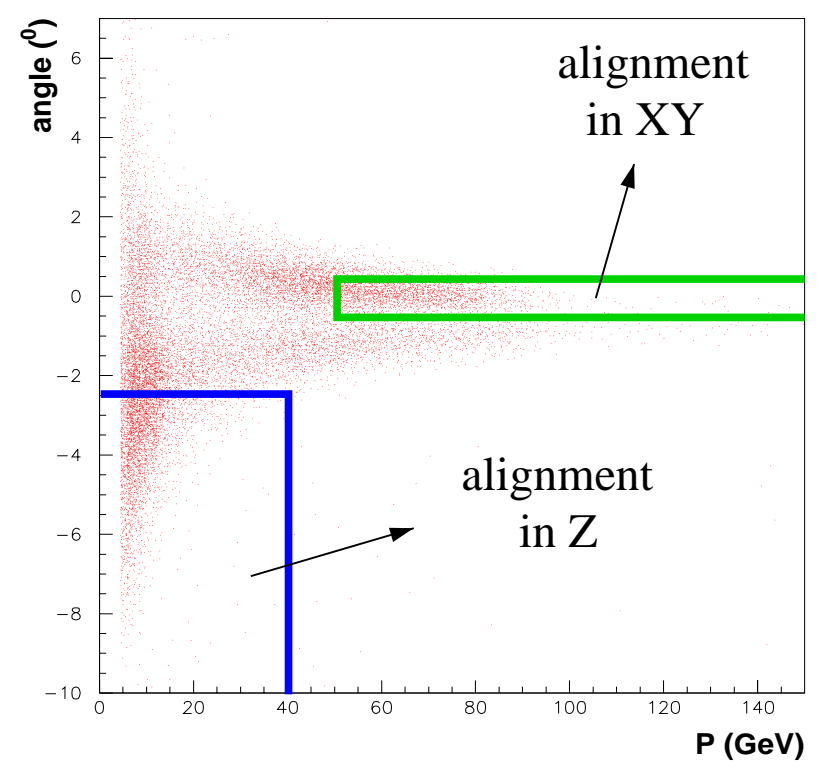

Figure 11: Angle-momentum regions used for the alignment in the $x y$ plane and the alignment in $z$.

Each of the two alignments is performed by minimizing the residuals on the position of a ladder. The residual is defined, in this case, as the difference between the predicted position of the hit and the measured position given by the response of a ladder. The predicted position of a hit is the extrapolation of the reference track to the theoretical plane of the ladder without including the hit in that ladder. The Kalman filter described in Section 3 is used to build the reference track with one or more hits in silicon and including the DC information. The Kalman filter is also used to calculate the hit prediction in the ladder. A $\chi^{2}$ minimization of the residuals for each detector determines the detector parameters.

\subsection{Alignment in the $x y$ plane}

The silicon ladders are almost contained in the $x y$ plane. Three parameters describe the position of a detector inside a plane: two shifts $\left(x_{0}\right.$ and $\left.y_{0}\right)$ and one angle $\theta$. But a silicon ladder provides only one coordinate (almost equivalent to the $y$ coordinate in the NOMAD reference frame, because the strips are nearly parallel to the $x$ axis, see Fig. 12), so we do not have any information about the shift $x_{0}$.

We make the assumption that the ladders are in the $x y$ plane and the muons are perpendicular to that plane. The error incurred by this assumption is negligible compared to the intrinsic resolution of the detectors $(\sim 5 \mu \mathrm{m})$.

The measured $y$ position needed to calculate $\chi^{2}$ is given by: 


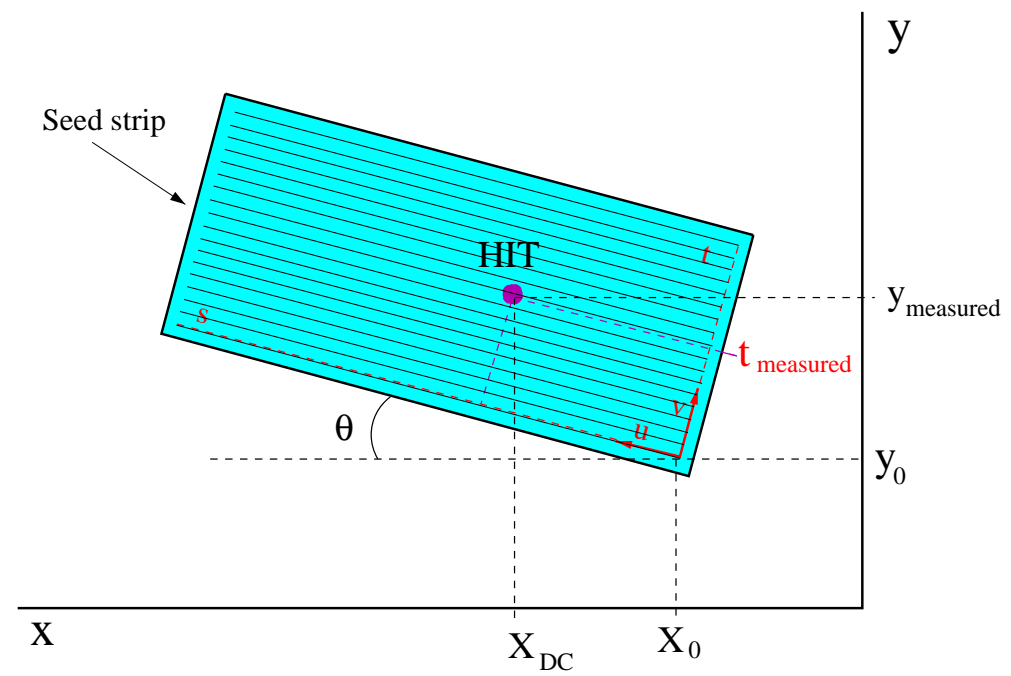

Figure 12: Alignment in the $x y$ plane.

$$
y_{\text {measured }}=y_{0}+s \cdot \underbrace{\sin \theta}_{u_{y}}+t \cdot \underbrace{\cos \theta}_{v_{y}},
$$

where

$$
\begin{gathered}
t=\text { strip } \cdot \text { PITCH } \\
s=(x_{D C}-x_{0}+t \cdot \underbrace{\sin \theta}_{v_{x}}) / \underbrace{\cos \theta}_{u_{x}},
\end{gathered}
$$

with $P I T C H=50 \mu \mathrm{m}$ and $x_{D C}$ the extrapolated $x$ position from the DC. If we assume small corrections to $y_{0}$ and $\theta\left(\delta y_{0}, \delta \theta\right)$ (to first order):

$$
y_{\text {measured }}^{\prime}=y_{\text {measured }}+\delta \mathbf{y}_{\mathbf{0}}+s \cdot \delta \theta .
$$

Minimizing the $\chi^{2}$ we get a system of two linear equations which can be easily solved.

\subsection{Alignment in $z$}

As we have demonstrated in sub-section 4.3, the alignment in $z$ requires high angle tracks in the $y z$ plane with respect to the $z$ axis. We have to choose the parameters to be corrected in such a way that the alignment in $z$ does not affect the previous alignment in $x y$ (Fig. 13):

- the angle $\gamma$ describes the rotation around the $y$ axis,

- the angle $\alpha$ describes the rotation around the axis defined by $\vec{u}$, and 
- the shift $z_{0}$ from the nominal ladder position.

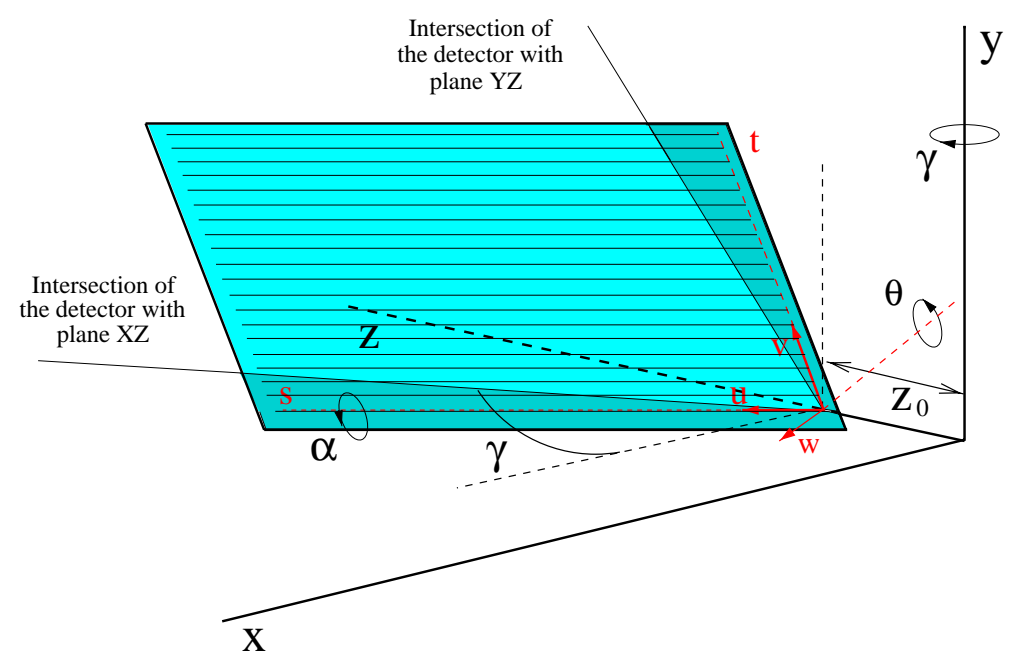

Figure 13: Definition of parameters for the alignment in $z$.

The angle $\theta$ is defined in Fig. 12. It describes the rotation inside the plane in which the ladder is contained around the axis defined by $\vec{w}$. The $z$ coordinate of a given point inside a ladder is

$$
z=z_{0}+s \cdot(\underbrace{\cos \theta \cdot \sin \gamma}_{u_{z}})+t \cdot(\underbrace{-\cos \alpha \cdot \sin \theta \cdot \sin \gamma+\sin \alpha \cdot \cos \gamma}_{v_{z}})
$$

where

$$
s=\left[x_{D C}-x_{0}+t \cdot(\underbrace{\cos \alpha \cdot \sin \theta \cdot \cos \gamma+\sin \alpha \cdot \sin \gamma}_{v_{x}})\right] / \underbrace{\cos \theta \cdot \cos \gamma}_{u_{x}} .
$$

If we introduce Eq. 104 into Eq. 103:

$$
z=z_{0}+\left(x_{D C}-x_{0}\right) \tan \gamma+t \frac{\sin \alpha}{\cos \gamma} .
$$

If a ladder was completely contained in the $x y$ plane, $\alpha$ and $\gamma$ would be 0 . Assuming that the angles $\alpha$ and $\gamma$ are small, and small corrections to $z_{0}, \alpha$ and $\gamma$, the change in the $z$ coordinate of the point would be:

$$
\delta z=\delta z_{0}+\left(x_{D C}-x_{0}\right) \frac{1}{\cos ^{2} \gamma} \delta \gamma+t \frac{\cos \alpha}{\cos \gamma} \delta \alpha
$$

The $y$ coordinate predicted by the cubic model for a given $z$ is: 


$$
y_{\text {pred }}=a+b z+c z^{2}+d z^{3} .
$$

For small corrections to $z$ given by Eq. 106 we get

$$
y_{\text {pred }}^{\prime}=y_{\text {pred }}+\frac{d y_{\text {pred }}}{d z} \cdot \delta z+\frac{1}{2} \frac{d^{2} y_{\text {pred }}}{d z^{2}} \cdot \delta z^{2}+\frac{1}{6} \frac{d^{3} y_{\text {pred }}}{d z^{3}} \cdot \delta z^{3}
$$

Minimizing the $\chi^{2}$ function, we get the desired corrections, $\delta z_{0}, \delta \alpha, \delta \gamma$ :

$$
\chi^{2}=\sum_{i=1}^{N}\left(\frac{y_{\text {pred }}^{\prime}\left(\delta z_{0}, \delta \alpha, \delta \gamma\right)-y_{\text {measured }}}{\sigma_{\text {res }}}\right)^{2} .
$$

\subsection{Alignment procedure}

The muons available are quite straight so most of them pass through the corresponding ladder (at the same height) for each plane (see Fig. 14). We define a set of ladders as the five ladders (one per plane) located at the same height.

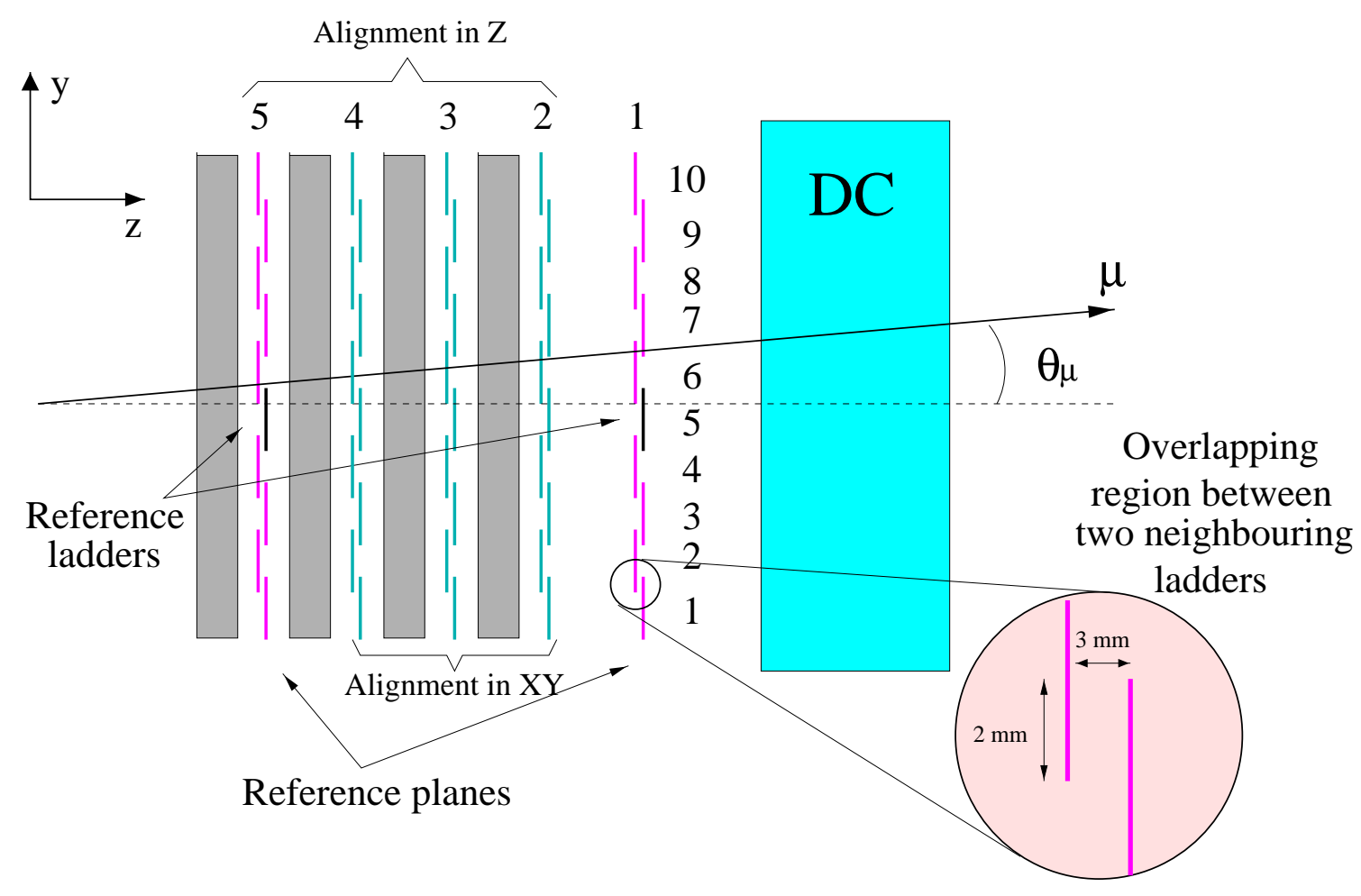

Figure 14: The alignment procedure.

We summarize the sequence of steps in which the alignment takes place:

- Global alignment ladder by ladder with respect to the DC. 
- Definition of the internal reference frame: ladder 5 in the center of the outermost planes (1, the closest to the DC, and 5, the furthest from the DC, see Fig. 14).

- Correction of $\theta$ for ladder 5 in plane 5 (relative angle between planes 1 and 5 ).

- Alignment of planes 1 and 5 by using the overlaps between two contiguous ladders.

- Iterative alignment in $x y$ of all the ladders in the inner planes (2-4).

- Iterative alignment in $z$ of all the ladders in planes 2-5.

\subsubsection{Alignment with DC}

To improve the $0.4 \mathrm{~mm}$ precision of the NOMAD-STAR mini-basket with respect to the rest of the NOMAD detector, we need to use the DC to determine a more precise location of NOMAD-STAR with respect to the NOMAD reference frame. The first step of the alignment consists in correcting the position of all the ladders by minimizing the residuals defined by the DC extrapolation and the hit in the corresponding ladder (Fig. 14). This procedure locates NOMAD-STAR in the NOMAD reference frame with a precision of around $120 \mu \mathrm{m}$.

\subsubsection{The internal reference frame}

To achieve an intrinsic resolution of around $5 \mu \mathrm{m}$ for an individual ladder from a relative alignment of $120 \mu \mathrm{m}$ with respect to the DC, the final alignment has to be completely internal, so we need to define an internal reference system for NOMAD-STAR.

The axes are defined in Fig. 15. The $x^{\prime}$ axis is given by $P_{1}$ and $P_{2}$, two points of ladder 5 in plane 1 (the closest to the DC). This ladder remains untouched after the alignment with the DC. The $z^{\prime}$ axis will be perpendicular to $x^{\prime}$ containing $P_{3}\left(\vec{r}_{0}\right.$ of ladder 5 in plane 5). Once $z^{\prime}$ is defined, we can move $P_{3}$ along the $z^{\prime}$ axis when performing the alignment. Finally, $y^{\prime}$ will be perpendicular to the other two axes. The strip pitch defines the scale of the $y^{\prime}$ axis, while the scale of the $z^{\prime}$ axis is given by the projection of the strip pitch to this axis given by muons (the $z^{\prime}$ scale has no meaning before the alignment). The scale of the $x^{\prime}$ axis is not defined but is not important because we cannot measure the $x$ coordinate with the silicon.

As we can see in Fig. 15, there exists a relative angle $\left(\theta_{\text {ref }}\right)$ between the reference ladders that can be corrected, keeping the internal reference frame invariant. The way to correct this angle is by using the iterative procedure explained in section 4.6.4 for the 5th ladder in each plane, allowing changes in $\theta_{\text {ref }}$ and $y_{0}$ for planes $2-4$ and changes only in $\theta_{\text {ref }}$ for plane 5 ( $y_{0}$ fixed, see 4.4). This step has to be done before the alignment by overlaps.

\subsubsection{Alignment by overlaps}

The way in which the internal reference frame has been defined in section 4.6.2 only works for muons crossing ladder 5 in planes 1 and 5 . To extend the internal reference system to the whole detector we must relate the positions of all the ladders in these reference planes 


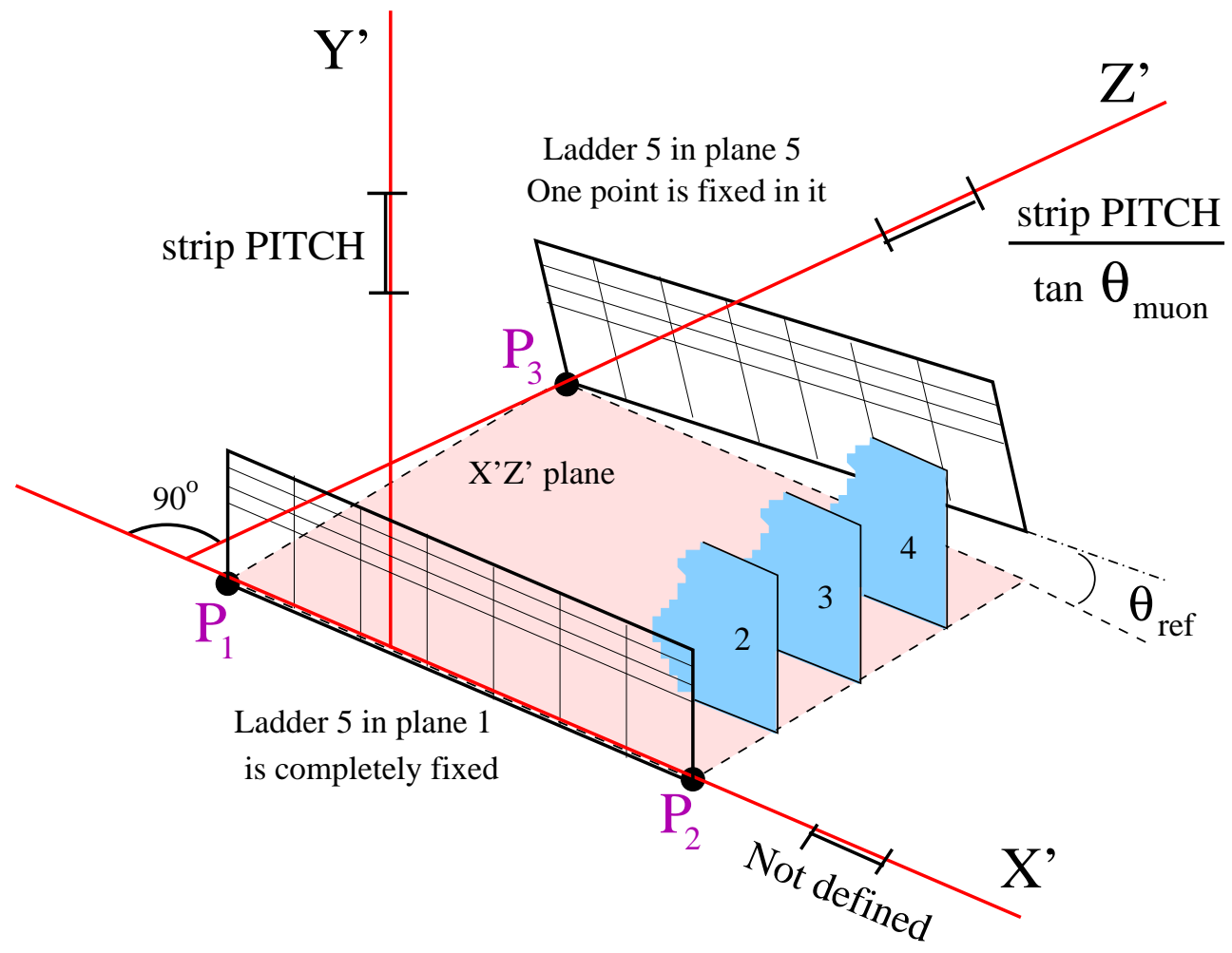

Figure 15: Definition of the internal reference system 
( 1 and 5 ). This is done by aligning the ladders in these planes taking ladder 5 as reference. The way to do it is by using the hits passing across the region where two contiguous ladders overlap (Fig. 14). The distance between these ladders in $z$ is only $3 \mathrm{~mm}$. In addition, multiple scattering can be neglected because there is no passive material between them. Therefore, tracks with only two hits (in both overlapped ladders) are sufficient to get a good alignment between them. The reference track is built with the hit in one of the ladders that overlap and the information coming from the DC. Residuals in the other overlapped ladder are minimized in order to achieve the relative alignment. If ladder 5 in planes 1 and 5 are the reference ladders, we start aligning ladder 6 and 4 using the overlaps 5-6 and 5-4 respectively. Afterwards overlaps 6-7 and 4-3 allow us to align ladders 7 and 3 , and so on.

\subsubsection{Iterative alignment}

This is the most complicated part of the alignment. It allows us to define the scale in $y^{\prime}$ for the inner planes and in $z^{\prime}$ for the whole detector. Once the outermost planes have been aligned, the alignment in $x y$ of the inner layers and the alignment in $z$ are performed in an iterative way (Fig. 14). Let us consider a set of ladders (5 ladders, one per plane, each one in the same position within a plane).

We only use tracks with hits in all planes. For the average of many tracks we have to correct the position of the ladder that makes the $\chi^{2}$ of the track worst, or equivalently, the one which gives the best $\chi^{2}$ when the hit is removed from the fit. We repeat this process until the $\chi^{2}$ is stable (when the change is less than $1 \%$ ). This operation has to be repeated for the ten sets of ladders, performing both the alignment in $x y$ and the alignment in $z$.

- The alignment in $x y$ (see section 4.4) is done first, using very straight muons. Only ladders in the inner planes (2-4) are allowed to be corrected in order to keep the internal reference frame invariant (see 4.6.2).

- The alignment in $z$ (see 4.5) requires high angle tracks. It involves planes 2-5. Plane 1 defines the origin.

One can also perform a cross-check to make sure that everything is consistent. Having aligned two contiguous sets of ladders independently to each other, we can check that the residuals are centered at zero using the overlaps between them for the inner planes.

\subsubsection{Alignment per detector}

The alignment per ladder makes the assumptions that the movements of individual detectors inside a ladder are negligible and that the survey error of $\sigma_{\text {survey }} \sim 4 \mu \mathrm{m}$ does not add significantly to the overall resolution. We attempted to perform detector-by-detector corrections. However, since the statistics for a single detector are small and there is not sufficient lever arm along the $5 \mathrm{~cm}$ length of the detector in the $x$ direction to make corrections in the angle $\theta$, we can only attempt to correct $y_{0}$ (see section 4.4 ):

$$
y_{\text {measured }}^{\prime}=y_{\text {measured }}+\delta y_{0}
$$


When we attempted to align the individual detectors inside a ladder, the corrections we found were of the order of 2 or 3 microns, compatible with the survey errors and showing no apparent internal movements. These corrections are not significant compared with the other contributions to the residuals, like the intrinsic resolution, multiple scattering and extrapolation error, so we have decided not to perform a systematic alignment detector-bydetector.

\subsection{Results}

Fig. 16 shows the residuals after the alignment for muons with high momentum $(p>50 G e V)$, and low angle $\left(\theta_{\text {muon }} \in[-0.5,0.5]^{\circ}\right)[28]$.

We have found the error in the residuals to be $9 \mu \mathrm{m}$ for the three inner planes (planes $2-4$ ) and a higher value of $12 \mu \mathrm{m}$ for the two outer planes (planes 1 and 5). This is an effect of the Kalman filter since in an inner plane we have information from both sides of the plane to predict the position of the hit. However, for an outermost plane, the information is only on one side. The error due to multiple scattering and the one related with the extrapolation is then larger in the case of the outermost planes.

\section{Impact parameter}

An example of the use of the vertex fit is the measurement of the impact parameter resolution of NOMAD-STAR [31]. In a $\nu_{\mu}$ charged current interaction, the $\mu^{-}$and hadronic jet come from the same point in space, and so if the $\mu^{-}$track is removed from the vertex fit, it should still point to the vertex that is now composed only of the hadronic jet. The impact parameter $(d)$ has been defined in section 2 as the projected signed distance of closest approach to the $\mu^{-}$from a $\nu_{\mu}$ charged current interaction to the vertex produced by the remaining hadronic jet. The impact parameter significance is the same quantity divided by the calculated error.

The procedure for measuring the impact parameter resolution of STAR uses both the vertex filter and the inverse filter. The first stage is to fit the $\mu^{-}$and hadronic jet into one vertex (the primary vertex). If there is an identified $\mu^{-}$in the vertex, it can then be removed from the vertex using the inverse filter. At this point the vertex position is now determined only by the hadronic jet. The $\mu^{-}$track can then be extrapolated to this new vertex position and the projected impact parameter can then be measured.

The results obtained for the impact parameter and impact parameter significance for the $\nu_{\mu}$ charged current interactions obtained from the NOMAD-STAR 1998 data set are shown in Fig. 17. The comparison of the data and $\nu_{\mu}$ charged current Monte Carlo distributions are in good agreement and show an RMS of approximately $52 \mu \mathrm{m}$ and an impact parameter significance with a fitted mean close to zero and width of 1.2. The non-Gaussian tail of the distribution is attributed to two track events with a small opening angle. Imposing that the two tracks have an opening angle of greater than 0.2 rad yields the bottom distributions shown in Fig. 17. The RMS of the impact parameter is now $36 \mu \mathrm{m}$ and the impact parameter significance is Gaussian with a width of 1.0, which indicates that the errors are being taken 
Plane 1



Plane 3

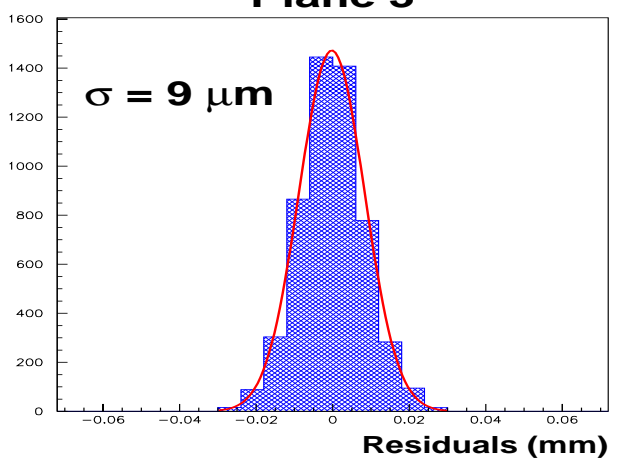

Plane 2

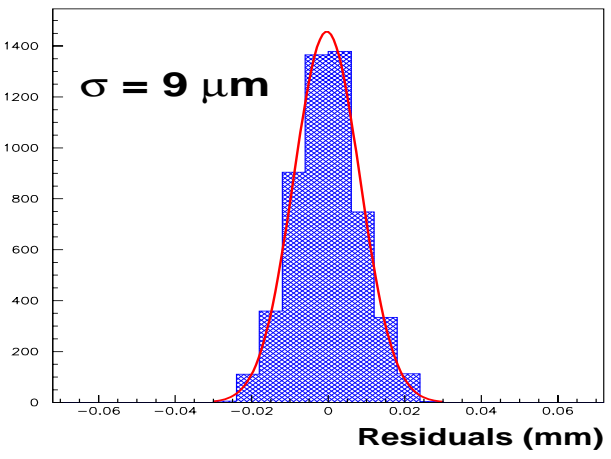

Plane 4

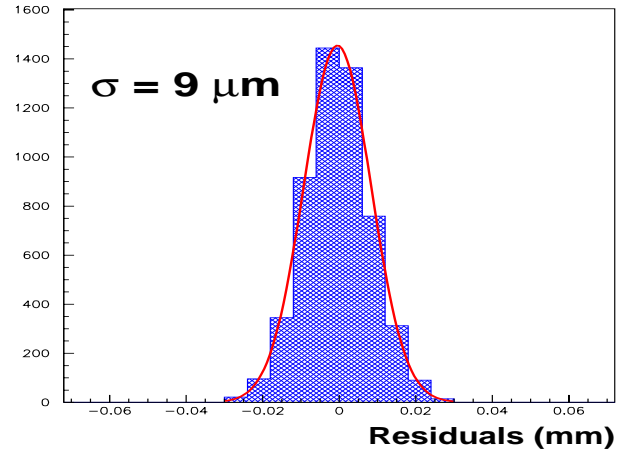

Plane 5

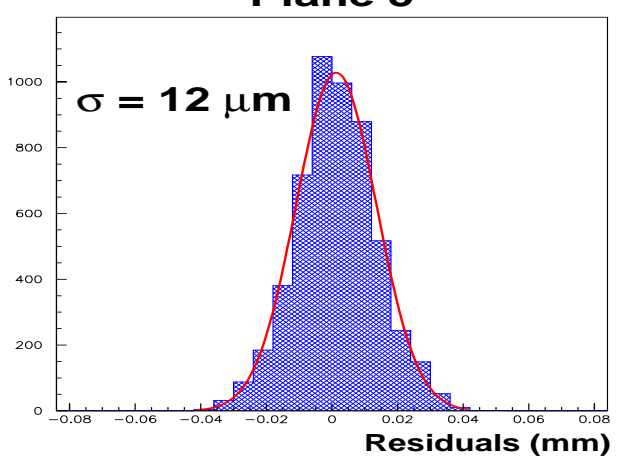

Figure 16: Distribution of residuals after the alignment. 
into account properly. Cutting out all two track events results in an impact parameter with RMS of $33 \mu \mathrm{m}$, which is close to the value of $28 \mu \mathrm{m}$ described in [3] and shown in Fig. 4 . Any further differences are probably also due to the limited lever arm of NOMAD-STAR. With only 5 silicon layers, it is frequent that there are only two or three hits that define a track, compared to the 10 silicon layers in the NAUSICAA design.

The impact parameter significance $\left(d_{s}\right)$ has quite a similar distribution to the NAUSICAA proposal, justifying cuts on this variable. If one would use this detector for $\nu_{\mu}\left(\nu_{e}\right) \leftrightarrow \nu_{\tau}$ oscillations, where the $\tau^{-}$decays to a $\mu^{-}$, one would obtain an exponential impact parameter distribution with an RMS around $62 \mu \mathrm{m}$ (Fig. 4). A cut at $d_{s}>4$ reduces the $\nu_{\mu} \mathrm{CC}$ background by 2 orders of magnitude. According to [3], a detector like NOMAD-STAR would have a $10 \%$ efficiency for $\tau$ detection when the $\tau$ decays to one charged particle while having a background rejection factor of more than $10^{6}$. Furthermore, one can mimic the $\nu_{\mu}\left(\nu_{e}\right) \leftrightarrow \nu_{\tau}$ signature in data by studying short lived particles like $K_{s}, \Lambda$ and charm particles. A search for such short-lived states is currently in progress.

\section{Conclusion}

NOMAD-STAR, a prototype silicon tracking detector installed inside the NOMAD magnet, can be used as an example of the application of Kalman filtering techniques for tracking and vertexing of particles. In the present paper we have shown the Kalman filter algorithms used for tracking particles from neutrino interactions in the NOMAD-STAR volume and for finding their associated vertices. An application of the tracking algorithms is the alignment of the NOMAD-STAR detector with through-going muons traversing it. In addition, the vertexing algorithms are used for the determination of the impact parameter of $\nu_{\mu}$ charged current interactions.

These algorithms have proven to be very effective in achieving alignment residuals between 9 and $12 \mu \mathrm{m}$ and an impact parameter distribution from $\nu_{\mu}$ charged current interactions with an RMS of $36 \mu \mathrm{m}$ when small opening angle events (less than $0.2 \mathrm{rad}$ ) for two-track primary vertices are removed. This encouraging result shows potential for a similar type of detector to measure $\nu_{\mu}\left(\nu_{e}\right) \leftrightarrow \nu_{\tau}$ signals with high efficiency.

Acknowledgements Funding is acknowledged from the EP Division at CERN; ARC and DISR (Australia). We would like to thank all the people that have made the NOMADSTAR detector possible: G. Baricchello, D.C. Daniels, L. Dumps, C. Gößling, D. Geppert, S. Geppert, W. Huta, J.M. Jiménez, J. Long, B. Lisowski, A. Lupi, K. Mühleman, J. Mulon, B. Schmidt, D. Steele, M. Stipčević, M. Veltri and D. Voillat, and the encouragement and support of all the NOMAD institutions. F.J.P. Soler is supported by a TMR Fellowship from the European Commission. J. Kokkonen acknowledges support from the Academy of Finland, the Magnus Ehrnrooth Foundation, the Foundation for the Commercial and Technical Sciences (KAUTE) and the Waldemar von Frenckell Foundation. 

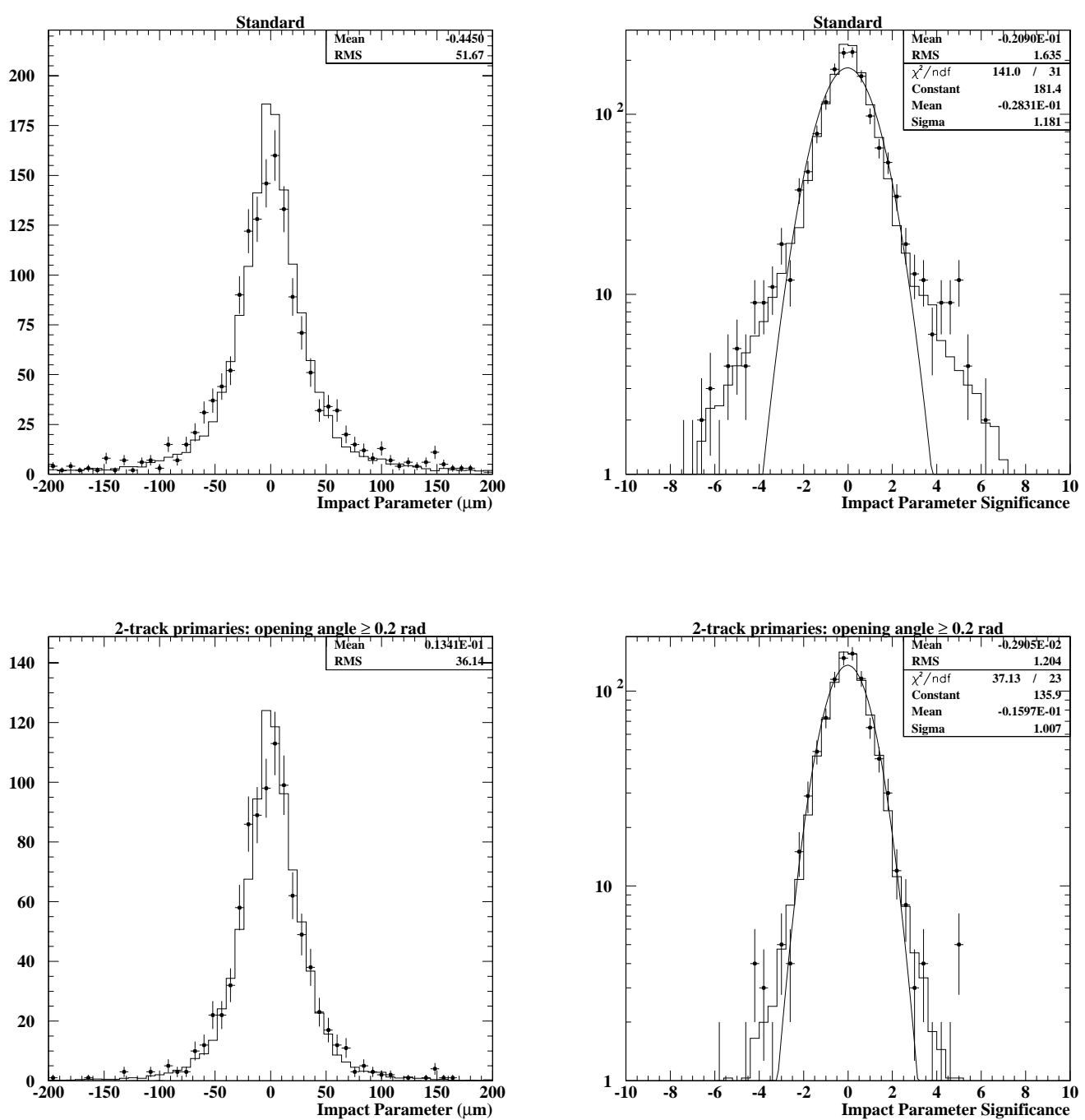

Figure 17: Impact parameter distribution (left) and impact parameter significance (right) of $\nu_{\mu}$ CC data (points) and Monte Carlo (histogram). The top figures are for all events with two or more tracks and the bottom figures are cutting out events with two tracks and an opening angle of less than $0.2 \mathrm{rad}$. 


\section{References}

[1] E.Eskut et al., CHORUS Collaboration, Nucl. Instr. and Meth. A401 (1997), 352; E.Eskut et al., CHORUS Collaboration, Phys. Lett. B434 (1998) 205.

[2] J. Altegoer et al., The NOMAD Collaboration, Nucl. Instr. and Meth. A404 (1998) 96; P. Astier et al., The NOMAD Collaboration, Physics Letters B453 (1999) 169.

[3] J.J. Gómez-Cadenas, J.A. Hernando and A. Bueno, Nucl. Instr. and Meth. A378 (1996) 196.

[4] J.J. Gómez-Cadenas and J.A. Hernando, Nucl. Instr. and Meth. A381 (1996) 223-235.

[5] A.S. Ayan et al., CERN-SPSC/97-5,SPSC/I213, March, 1997.

[6] O. Toker, S. Masciocchi, E. Nygård, A. Rudge, P. Weilhammer, Nucl. Instr. and Meth. A340 (1994) 572.

[7] P.P. Allport et al., Nucl. Instr. and Meth. A310 (1991) 155.

[8] G. Baricchello et al., Nucl. Instr. and Meth. A413 (1998) 17.

[9] G. Baricchello et al., Nucl. Instr. and Meth. A419 (1998) 1.

[10] R.E. Kalman, J. Basic Eng. 82 (1960) 35; R.E. Kalman and R.S. Bucy, J. Basic Eng. 83 (1961) 95.

[11] R. Frühwirth, Nucl. Instr. and Meth. A262 (1987) 444.

[12] P. Billoir, Nucl. Instr. and Meth. 225 (1984) 352.

[13] P. Billoir, R. Frühwirth and M. Regler, Nucl. Instr. and Meth. A241 (1985) 115.

[14] L. Stanco, Computer Physics Communications 57 (1989) 380.

[15] P. Billoir and S. Qian, Nucl. Instr. and Meth. A294 (1990) 219; P. Billoir and S. Qian, Nucl. Instr. and Meth. A295 (1990) 492.

[16] P. Billoir and S. Qian, Nucl. Instr. and Meth. A311 (1992) 139.

[17] E. Calligarich, R. Dolfini, M. Genoni and A. Rotondi, Nucl. Instr. and Meth. A311 (1992) 151.

[18] V. Innocente and E. Nagy, Nucl. Instr. and Meth. A324 (1993) 297.

[19] E.J. Wolin and L.L. Ho, Nucl. Instr. and Meth. A329 (1993) 493.

[20] R. Luchsinger and C. Grab, Computer Physics Communications 76 (1993) 263.

[21] P. Astier et al., Nucl. Instr. and Meth. A450 (2000) 138. 
[22] V.E. Kuznetsov, Nucl. Phys. B (Proc. suppl.) 78 (1999) 287.

[23] G.Z. Molière, Z. Naturforsch. 2a (1947) 133; Z. Naturforsch. 3a (1948), 78.

[24] H.A. Bethe, Phys. Rev. 89 (1953), 1256.

[25] G.R. Lynch and O.I. Dahl, Nucl. Instr. and Meth. B58 (1991) 6.

[26] GEANT, CERN Program Library Long Writeup W5013, (1994), 234.

[27] Particle Data Group, Eur. Phys. J. C (2000) 163.

[28] A. Cervera-Villanueva, Nucl. Instr. and Meth. A447 (2000) 100.

[29] A. Cervera-Villanueva et al., Survey of the Frames for STAR, NOMAD Internal Note, NOMAD-MEMO-97-48, 18 December 1997.

[30] J. Altegoer et al., Nucl. Instr. and Meth. A428 (1999) 299.

[31] F.J.P. Soler, "Silicon Detectors for Neutrino Physics Experiments", 5th International Conference on Position Sensitive Detectors, University College, London, September 1999. To be published in Nuclear Instruments and Methods. 